\title{
An overview of the methods for deriving recurrence relations for T-matrix calculation
}

\author{
Adrian Doicu ${ }^{\mathrm{a}}$, Thomas Wriedt ${ }^{\mathrm{b}, *}$, Naima Khebbache ${ }^{\mathrm{b}}$ \\ ${ }^{a}$ Remote Sensing Technology Institute, German Aerospace Centre, Oberpfaffenhofen, Wessling, Germany \\ ${ }^{\mathrm{b}}$ Leibnitz Institute for Materials Engineering IWT, University of Bremen, Bremen, Germany
}

\section{A R T I C L E I N F O}

\section{Article history:}

Received 9 July 2018

Revised 20 November 2018

Accepted 20 November 2018

Available online 22 November 2018

\begin{abstract}
A B S T R A C T
In this paper, we present a consistent analysis of the methods dealing with the derivation of recurrence relations for calculation of the T-matrix. Central and forward recurrence relations are obtained in the framework of the invariant embedding T-matrix method, the matrix Riccati equation method, and the superposition T-matrix method. The accuracies and efficiencies of the central and forward recurrence schemes are analyzed, and some implementation issues related to the improvement of the numerical accuracy and to the problem of overcoming of overflow errors are discussed.
\end{abstract}

(c) 2018 Published by Elsevier Ltd.

\section{Introduction}

The null-field method (otherwise known as the extended boundary condition method) is one of the most efficient methods for analyzing the electromagnetic scattering by large and highly non-spherical particles. The method, which was initially proposed by Watermann [1,2] and later developed by Barber and Hill [3], and Mishchenko et al. [4], computes the $T$ matrix relating the scattered and incident field coefficients by making use on the nullfield equation for the total field inside the particle. Despite its wide range of applicability, the method suffers from numerical problems regarding convergence and loss of accuracy. The reason is that the null-field equation is a Fredholm integral equation of the first kind, which is known to be (severely) ill-posed. A number of methods have been proposed to improve the numerical stability in computations for large particles with extreme geometries. Here we include methods (i) dealing with the numerical stability of the inversion process [2,5-8], (ii) methods based on accurate computations of the elements of the so-called $Q$ matrices [9-15], (iii) methods using extended- and the multiple-precision floating-point variables $[9,16]$, and (iv) methods relying on formal modifications of the single spherical coordinate-based null-field method [17-22].

The invariant embedding T-matrix method, proposed by Johnson [23], is an efficient tool for analyzing the electromagnetic scattering by large and highly non-spherical particles. The method originates from an electromagnetic volume integral equation in spherical coordinates, and is based on the equivalence between

\footnotetext{
* Corresponding author.

E-mail address: thw@iwt.uni-bremen.de (T. Wriedt).
}

the scattering by a non-spherical particle and the scattering by the inhomogeneous circumscribed sphere. Essentially, Johnson derived a recurrence relation for the $T$ matrix by applying the invariant embedding procedure on the discrete form of the volume integral equation; the $\mathrm{T}$ matrix is computed recursively by growing the scattering volume incrementally in a shell-by shell manner, while the initial $\mathrm{T}$ matrix is computed by means of the separation of variables method [24] or the null-field method [25]. As the volume integral equation is a Fredholm integral equation of the second kind, the method does not suffer from ill-posedness, and its numerical performances are really remarkable. Excellent numerical results have been obtained for large spheroids and cylinders (size parameters up to 300), cylinders with large aspect ratios (20:1), inhomogeneous particles, and two-particle systems [24]. Accurate simulations of the optical properties of randomly oriented ice crystals of various shapes including hexagonal columns, hollow columns, droxtals, bullet rosettes and aggregates with size parameters up to 150 have been reported in Ref. [26].

In Ref. [27], we revised the theoretical foundation of the invariant embedding T-matrix method and derived a matrix Riccati equation for the $\mathrm{T}$ matrix. By directly applying the invariant embedding procedure to the continuous form of the Fredholm integral equation we rediscovered Johnson's recurrence relation, while by using the modified Davison-Maki method for solving the matrix Riccati equation we found a new recurrence relation forTmatrix calculation. However, because in the latter case, the method is based on a first-order Taylor approximation to the matrix exponential, the accuracy of the T matrix is rather low. In Ref. [27] we also established the connection between the invariant embedding T-matrix method and the superposition T-matrix method. In fact, 
we proved that a recurrence relation derived in the framework of the superposition T-matrix method is analytically equivalent to Johnson's recurrence relation.

From a theoretical point of view, Johnson's derivation has an apparent weaknesses, which we now discuss. Let $f(r)$ be a function which is not defined at $r=b$ but has the left and right limits $f\left(b_{-}\right)$ and $f\left(b_{+}\right)$, respectively. A quadrature formula for computing the integral $\int_{a}^{b} f(r) \mathrm{d} r$, based say on the trapezoidal rule, reads as

$I=\int_{a}^{b} f(r) \mathrm{d} r \approx \sum_{k=0}^{n-1} w_{k} f\left(r_{k}\right)+\frac{1}{2} \Delta r\left[f\left(r_{n-1}\right)+f\left(b_{-}\right)\right]$,

where $\Delta r=(b-a) / n, r_{k}=a+k \Delta r, w_{0}=w_{n-1}=\Delta r / 2$, and $w_{k}=$ $\Delta r$ for $k=1, ., n-2$. In the framework of the invariant embedding T-matrix method, Johnson observed that more stable results are obtained if in Eq. (1), $f\left(b_{-}\right)$is replaced by

$f(b)=\frac{1}{2}\left[f\left(b_{-}\right)+f\left(b_{+}\right)\right]$,

and mentioned that "We do not, at present, have a good explanation for this; however, we assume that an explanation can be found and in the meantime we pragmatically use the method that gives the best results". Actually, the replacement $f\left(b_{-}\right) \rightarrow f(b)$ can be explained as follows. Consider the following integrals and the corresponding quadrature formulas:

$I_{\varepsilon-}=\int_{a}^{b-\varepsilon} f(r) \mathrm{d} r \approx \sum_{k=0}^{n-1} w_{k} f\left(r_{k}\right)+\frac{1}{2}(\Delta r-\varepsilon)\left[f\left(r_{n-1}\right)+f(b-\varepsilon)\right]$,

$I_{\varepsilon+}=\int_{a}^{b+\varepsilon} f(r) \mathrm{d} r \approx \sum_{k=0}^{n-1} w_{k} f\left(r_{k}\right)+\frac{1}{2}(\Delta r+\varepsilon)\left[f\left(r_{n-1}\right)+f(b+\varepsilon)\right]$,

where $\Delta r, r_{k}$, and $w_{k}$ are defined as above. Then, taking into account that

$I=\frac{1}{2} \lim _{\varepsilon \rightarrow 0}\left(I_{\varepsilon-}+I_{\varepsilon+}\right)$,

we find

$I \approx \sum_{k=0}^{n-1} w_{k} f\left(r_{k}\right)+\frac{1}{2} \Delta r\left[f\left(r_{n-1}\right)+f(b)\right]$

with $f(b)$ as in Eq. (2). Thus, the replacement $f\left(b_{-}\right) \rightarrow f(b)$ seems to be justified, and it is apparent that the same arguments hold for the right-endpoint quadrature formula:

$I=\int_{a}^{b} f(r) \mathrm{d} r \approx \sum_{k=1}^{n-1} \Delta r f\left(r_{k}\right)+\Delta r f\left(b_{-}\right)$.

On the other hand, if the point of discontinuity $r=b$ is an interior point of the interval $[a, c]$ with $c>b$, then the contribution of this point to the quadrature sum (1) is as in Eq. (2).

In the framework of the invariant embedding T-matrix method, the point of discontinuity is the point at which the radial matrix Green function (see Eq. (19) below) is not defined. The recurrence relations in Ref [27]. have been obtained by treating the point of discontinuity as the right endpoint of an integration interval. Nevertheless, from a theoretical point of view, it is interesting to see which are the recurrence relations when the point of discontinuity is an interior point of an integration interval. In this review paper we extend the analysis of Ref. [27] to fulfill this desideratum. According to the terminology used in the finite-difference method, a recurrence relation in which the point of discontinuity is the midpoint of an integration interval will be called a central recurrence relation, while the name forward recurrence relation will be used when the point of discontinuity is the right endpoint. For the sake of completeness and the convenience of the reader we borrow some parts of the derivation outlined in Ref. [27].

\section{The volume integral equation method}

In the volume integral equation method it is assumed that the particle is entirely contained within a sphere of radius $R$ and interior $D$, and is completely described by specifying the relative refractive index $m_{\mathrm{r}}(\mathbf{r})$ as a function of the position vector $\mathbf{r}$ in $D$ (Fig. 1a). In this section we derive

1. a matrix-form representation for the electromagnetic volume integral equation in spherical coordinates,

2. a Fredholm integral equation of the second kind for the radial amplitude vector by using the representation of the free-space dyadic Green function in terms of spherical vector wave functions,

3. two-terms recurrence relations for T-matrix calculation by applying the invariant embedding procedure,

4. a matrix Riccati equation for the T matrix, and subsequently, two-terms recurrence relations for T-matrix calculation by using the modified Davison-Maki method for integrating the matrix Riccati equation.

For simplicity reasons we restrict our analysis to axisymmetric particles.

\subsection{The electromagnetic volume integral equation in spherical coordinates}

Throughout our analysis, we assume the harmonic time dependence $\exp (-\mathrm{j} \omega t)$ for the fields, where $\mathrm{j}=\sqrt{-1}, t$ is time, and $\omega$ is the angular frequency. For the geometry considered in Fig. 1a, the total (electric) field $\mathbf{E}(\mathbf{r})$ satisfies the volume integral equation

$\mathbf{E}(\mathbf{r})=\mathbf{E}_{0}(\mathbf{r})+\int_{D} \chi\left(\mathbf{r}^{\prime}\right) \overline{\mathbf{G}}\left(\mathbf{r}, \mathbf{r}^{\prime}\right) \cdot \mathbf{E}\left(\mathbf{r}^{\prime}\right) \mathrm{d}^{3} \mathbf{r}^{\prime}$,

where $\mathbf{E}_{0}(\mathbf{r})$ is the incident field, $\overline{\mathbf{G}}\left(\mathbf{r}, \mathbf{r}^{\prime}\right)$ is the dyadic Green function,

$\chi(\mathbf{r})=k_{0}^{2}\left[m_{\mathrm{r}}^{2}(\mathbf{r})-1\right]$,

$k_{0}$ is the wavenumber in the host medium, and $m_{\mathrm{r}}$ is the relative refractive index of the particle. The dyadic Green function $\overline{\mathbf{G}}$ can be expressed as

$\overline{\mathbf{G}}\left(\mathbf{r}, \mathbf{r}^{\prime}\right)=\overline{\mathbf{G}}_{0}\left(\mathbf{r}, \mathbf{r}^{\prime}\right)-\frac{1}{k_{0}^{2}} \delta\left(\mathbf{r}-\mathbf{r}^{\prime}\right) \widehat{\mathbf{r}} \otimes \widehat{\mathbf{r}}^{\prime}$,

where $\widehat{\mathbf{r}}$ is the radial unit vector, $\otimes$ stays for the dyadic product, $\overline{\mathbf{G}}_{0}\left(\mathbf{r}, \mathbf{r}^{\prime}\right)$ is the free-space dyadic Green function possessing the representation

$$
\begin{aligned}
& \overline{\mathbf{G}}_{0}\left(\mathbf{r}, \mathbf{r}^{\prime}\right) \\
& =\frac{\mathrm{j} k_{0}}{\pi} \sum_{m n}\left\{\begin{array}{r}
\mathbf{M}_{m n}^{3}\left(k_{0} \mathbf{r}\right) \otimes \mathbf{M}_{-m n}^{1}\left(k_{0} \mathbf{r}^{\prime}\right)+\mathbf{N}_{m n}^{3}\left(k_{0} \mathbf{r}\right) \otimes \mathbf{N}_{-m n}^{1}\left(k_{0} \mathbf{r}^{\prime}\right), \\
r>r^{\prime} \\
\mathbf{M}_{m n}^{1}\left(k_{0} \mathbf{r}\right) \otimes \mathbf{M}_{-m n}^{3}\left(k_{0} \mathbf{r}^{\prime}\right)+\mathbf{N}_{m n}^{1}\left(k_{0} \mathbf{r}\right) \otimes \mathbf{N}_{-m n}^{3}\left(k_{0} \mathbf{r}^{\prime}\right), \\
r<r^{\prime}
\end{array},\right.
\end{aligned}
$$

$\mathbf{M}_{m n}^{1}, \mathbf{N}_{m n}^{1}$ and $\mathbf{M}_{m n}^{3}, \mathbf{N}_{m n}^{3}$ are the regular and radiating spherical vector wave functions, respectively, and the double sum should be understood as

$$
\sum_{m=-\infty}^{\infty} \sum_{n=\max (|m|, 1)}^{\infty}
$$




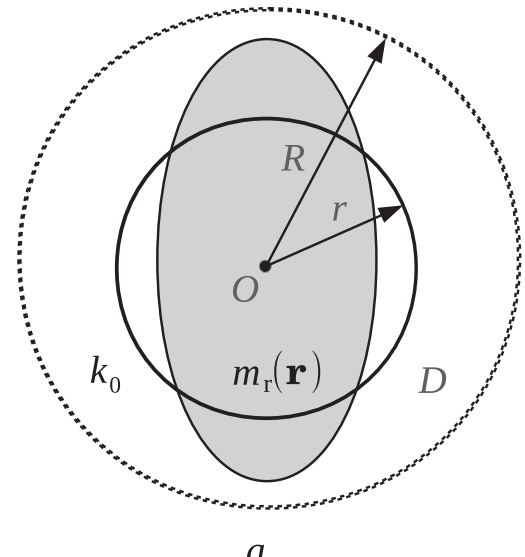

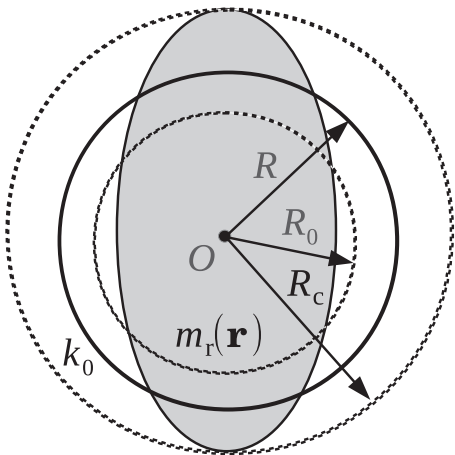

$b$

Fig. 1. Geometry of the scattering problem (a), and illustration of the invariant embedding T-matrix method (b).

In the following, we represent a vector $\mathbf{x}$ in the spherical basis $(\widehat{\mathbf{r}}, \widehat{\boldsymbol{\theta}}, \widehat{\boldsymbol{\varphi}})$ as

$\mathbf{x}=x_{r} \widehat{\mathbf{r}}+x_{\theta} \widehat{\boldsymbol{\theta}}+x_{\varphi} \widehat{\boldsymbol{\varphi}}=\left[\begin{array}{lll}\widehat{\mathbf{r}} & \widehat{\boldsymbol{\theta}} & \widehat{\boldsymbol{\varphi}}\end{array}\right] \times$,

where

$\mathrm{x}=\left[\begin{array}{l}x_{r} \\ x_{\theta} \\ x_{\varphi}\end{array}\right]$

is the column vector associated to the vector $\mathbf{x}$. Furthermore, with $\widehat{\mathbf{\Omega}}$ being a unit direction characterized by the polar angles $\theta$ and $\varphi$, i.e., $\boldsymbol{\Omega}=\widehat{\boldsymbol{\Omega}}(\theta, \varphi)$, we define the $3 \times 3$ matrix

$$
\begin{aligned}
\mathrm{Y}_{m n}(\widehat{\boldsymbol{\Omega}}) & =\frac{1}{\sqrt{2 n(n+1)}} \mathrm{e}^{\mathrm{j} m \varphi} \\
& \times\left[\begin{array}{ccc}
0 & 0 & \sqrt{n(n+1)} P_{n}^{|m|}(\cos \theta) \\
\mathrm{j} m \pi_{n}^{|m|}(\theta) & \tau_{n}^{|m|}(\theta) & 0 \\
-\tau_{n}^{|m|}(\theta) & \mathrm{j} m \pi_{n}^{|m|}(\theta) & 0
\end{array}\right],
\end{aligned}
$$

where $P_{n}^{|m|}(\cos \theta)$ are the normalized associated Legendre functions, $\tau_{n}^{|m|}(\theta)=\mathrm{d} P_{n}^{|m|}(\cos \theta) / \mathrm{d} \theta$ and $\pi_{n}^{|m|}(\theta)=P_{n}^{|m|}(\cos \theta) / \sin \theta$, and the $3 \times 2$ matrix

$\mathrm{X}_{n}^{1,3}(r)=\left[\begin{array}{cc}z_{n}^{1,3}\left(k_{0} r\right) & 0 \\ 0 & \frac{\left[k_{0} r z_{n}^{1,3}\left(k_{0} r\right)\right]^{\prime}}{k_{0} r} \\ 0 & \sqrt{n(n+1)} \frac{z_{n}^{1,3}\left(k_{0} r\right)}{k_{0} r}\end{array}\right]$,

where $z_{n}^{1}\left(k_{0} r\right)=j_{n}\left(k_{0} r\right)$ are the spherical Bessel functions and $z_{n}^{3}\left(k_{0} r\right)=h_{n}\left(k_{0} r\right)$ are the spherical Hankel functions. For the regular and radiating spherical vector wave functions, we will use the notation $\left(J_{n}(r)\right.$ should not be confused with the cylindrical Bessel functions)

$\mathrm{X}_{n}^{1}(r)=\mathrm{J}_{n}(r)$ and $\mathrm{X}_{n}^{3}(r)=\mathrm{H}_{n}(r)$.

In terms of these matrices, we find that

$\mathrm{Y}_{m n}(\widehat{\boldsymbol{\Omega}}) \mathrm{X}_{n}^{1,3}(r)=\left[\begin{array}{cc}M_{m n r}^{1,3}\left(k_{0} \mathbf{r}\right) & N_{m n r}^{1,3}\left(k_{0} \mathbf{r}\right) \\ M_{m n}^{1,3}\left(k_{0} \mathbf{r}\right) & N_{m n}^{1,3}\left(k_{0} \mathbf{r}\right) \\ M_{m n \varphi}^{1,3}\left(k_{0} \mathbf{r}\right) & N_{m n \varphi}^{1,3}\left(k_{0} \mathbf{r}\right)\end{array}\right]$,

where $M_{m n r}^{1,3}, M_{m n \theta}^{1,3}$ and $M_{m n \varphi}^{1,3}$ are the components of the vector $\mathbf{M}_{m n}^{1,3}$ in the spherical basis $(\widehat{\mathbf{r}}, \widehat{\boldsymbol{\theta}}, \widehat{\boldsymbol{\varphi}})$. Consequently, for say, $r>r^{\prime}$, the dyadic product in Eq. (11) can be expressed in tensor form representation as

$\mathbf{M}_{m n}^{3}\left(k_{0} \mathbf{r}\right) \otimes \mathbf{M}_{-m n}^{1}\left(k_{0} \mathbf{r}^{\prime}\right)+\mathbf{N}_{m n}^{3}\left(k_{0} \mathbf{r}\right) \otimes \mathbf{N}_{-m n}^{1}\left(k_{0} \mathbf{r}^{\prime}\right)$

$$
=\left[\begin{array}{lll}
\widehat{\mathbf{r}} & \widehat{\boldsymbol{\theta}} & \widehat{\boldsymbol{\varphi}}
\end{array}\right] \mathrm{Y}_{m n}(\widehat{\boldsymbol{\Omega}}) \mathrm{H}_{n}(r) J_{n}^{T}\left(r^{\prime}\right) \mathbf{Y}_{-m n}^{T}\left(\widehat{\boldsymbol{\Omega}}^{\prime}\right)\left[\begin{array}{c}
\widehat{\mathbf{r}}^{\prime} \\
\widehat{\boldsymbol{\theta}}^{\prime} \\
\widehat{\boldsymbol{\varphi}}^{\prime}
\end{array}\right],
$$

yielding

$$
\begin{aligned}
& \overline{\mathbf{G}}_{0}\left(\mathbf{r}, \mathbf{r}^{\prime}\right) \cdot \mathbf{E}\left(\mathbf{r}^{\prime}\right) \\
& =\frac{\mathrm{j} k_{0}}{\pi}\left[\begin{array}{lll}
\widehat{\mathbf{r}} & \widehat{\boldsymbol{\theta}} & \widehat{\boldsymbol{\varphi}}
\end{array}\right] \sum_{m n} \mathrm{Y}_{m n}(\widehat{\boldsymbol{\Omega}}) \mathrm{H}_{n}(r) J_{n}^{T}\left(r^{\prime}\right) \mathrm{Y}_{-m n}^{T}\left(\widehat{\boldsymbol{\Omega}}^{\prime}\right) \mathrm{E}\left(\mathbf{r}^{\prime}\right),
\end{aligned}
$$

where $E(\mathbf{r})$ is the column vector corresponding to $\mathbf{E}(\mathbf{r})$.

Inserting Eq. (10) in Eq. (8), and using the result of Eq. (16), we obtain the following matrix-form representation for the volume integral Eq. (8):

$$
\overline{\mathrm{E}}(\mathbf{r})=\mathrm{E}_{0}(\mathbf{r})+\sum_{m n} \mathrm{Y}_{m n}(\widehat{\boldsymbol{\Omega}}) \int_{D} \chi\left(\mathbf{r}^{\prime}\right) \mathrm{G}_{n}\left(r, r^{\prime}\right) \mathrm{Y}_{-m n}^{T}\left(\widehat{\boldsymbol{\Omega}}^{\prime}\right) \mathrm{Z}\left(\mathbf{r}^{\prime}\right) \overline{\mathrm{E}}\left(\mathbf{r}^{\prime}\right) \mathrm{d}^{3} \mathbf{r}^{\prime},
$$

where the column vector $\bar{E}(\mathbf{r})$ is defined through the relation $\mathrm{E}(\mathbf{r})=\mathrm{Z}(\mathbf{r}) \overline{\mathrm{E}}(\mathbf{r})$, with

$\mathrm{Z}(\mathbf{r})=\left[\begin{array}{ccc}1 / m_{\mathrm{r}}^{2}(\mathbf{r}) & 0 & 0 \\ 0 & 1 & 0 \\ 0 & 0 & 1\end{array}\right]$

$E_{0}(\mathbf{r})$ is the column vector corresponding to the incident field $\mathbf{E}_{0}(\mathbf{r})$, and $G_{n}\left(r, r^{\prime}\right)$ is the $3 \times 3$ (radial) matrix Green function given by [23]

$\mathrm{G}_{n}\left(r, r^{\prime}\right)=\frac{\mathrm{j} k_{0}}{\pi} \begin{cases}\mathrm{H}_{n}(r) J_{n}^{T}\left(r^{\prime}\right), & r>r^{\prime} \\ J_{n}(r) \mathrm{H}_{n}^{T}\left(r^{\prime}\right), & r<r^{\prime}\end{cases}$

The scattered field, defined by

$\mathbf{E}_{\mathbf{s}}(\mathbf{r})=\int_{D} \chi\left(\mathbf{r}^{\prime}\right) \overline{\mathbf{G}}_{0}\left(\mathbf{r}, \mathbf{r}^{\prime}\right) \cdot \mathbf{E}\left(\mathbf{r}^{\prime}\right) \mathrm{d}^{3} \mathbf{r}^{\prime}, \quad \mathbf{r} \in \mathbb{R}^{3} \backslash \bar{D}$,

where $\bar{D}=D \cup \partial D$ and $\partial D$ is the boundary of $D$, is expanded as (cf. Eq. (14))

$$
\begin{aligned}
\mathbf{E}_{\mathbf{s}}(\mathbf{r}) & =\sum_{m n} s_{m n}^{M} \mathbf{M}_{m n}^{3}\left(k_{0} \mathbf{r}\right)+s_{m n}^{N} \mathbf{N}_{m n}^{3}\left(k_{0} \mathbf{r}\right) \\
& =\left[\begin{array}{lll}
\widehat{\mathbf{r}} & \widehat{\boldsymbol{\theta}} & \widehat{\boldsymbol{\varphi}}
\end{array}\right] \sum_{m n} \mathbf{Y}_{m n}(\widehat{\boldsymbol{\Omega}}) \mathrm{H}_{n}(r)\left[\begin{array}{c}
s_{m n}^{M} \\
s_{m n}^{T}
\end{array}\right],
\end{aligned}
$$

in which case, we find that the column vector $E_{s}(\mathbf{r})$ corresponding to $\mathbf{E}_{\mathrm{s}}(\mathbf{r})$ is 
$\mathrm{E}_{\mathbf{s}}(\mathbf{r})=\sum_{m n} \mathbf{Y}_{m n}(\widehat{\boldsymbol{\Omega}}) \mathrm{H}_{n}(r) \mathbf{s}_{m n}$,

with

$\mathrm{s}_{m n}=\frac{\mathrm{j} k_{0}}{\pi} \int_{D} \chi(\mathbf{r}) J_{n}^{T}(r) \mathrm{Y}_{-m n}^{T}(\widehat{\boldsymbol{\Omega}}) \mathrm{Z}(\mathbf{r}) \overline{\mathrm{E}}(\mathbf{r}) \mathrm{d}^{3} \mathbf{r}$

and $s_{m n}=\left[s_{m n}^{M}, s_{m n}^{N}\right]^{T}$. Similarly, the incident field is expanded as

$\mathbf{E}_{0}(\mathbf{r})=\sum_{m n} a_{m n}^{M} \mathbf{M}_{m n}^{1}\left(k_{0} \mathbf{r}\right)+a_{m n}^{N} \mathbf{N}_{m n}^{1}\left(k_{0} \mathbf{r}\right)$,

and we have (cf. Eq. (14))

$\mathrm{E}_{0}(\mathbf{r})=\sum_{m n} \mathbf{Y}_{m n}(\widehat{\boldsymbol{\Omega}}) \mathrm{J}_{n}(r) \mathbf{a}_{m n}$,

where $a_{m n}=\left[a_{m n}^{M}, a_{m n}^{N}\right]^{T}$. The expansion coefficients of the scattered field $s_{m n}$ are related to the expansion coefficients of the incident field $a_{m n}$ through the T-matrix equation

$\mathrm{s}_{m n}=\sum_{m^{\prime} n^{\prime}} \mathrm{T}_{m n, m^{\prime} n^{\prime}} \mathrm{a}_{m^{\prime} n^{\prime}}$,

with

$\mathrm{T}_{m n, m^{\prime} n^{\prime}}=\left[\begin{array}{ll}\mathrm{T}_{m n, m^{\prime} n^{\prime}}^{11} & \mathrm{~T}_{m n, m^{\prime} n^{\prime}}^{12} \\ \mathrm{~T}_{m n, m^{\prime} n^{\prime}}^{21} & \mathrm{~T}_{m n, m^{\prime} n^{\prime}}^{22}\end{array}\right]$

2.2. The Fredholm integral equation for the radial amplitude vector

We define the radial amplitude vector $f_{m n}(r)$ by [23]

$\mathrm{f}_{m n}(r)=r^{2} \int_{4 \pi} \chi(\mathbf{r}) \mathrm{Y}_{-m n}^{T}(\widehat{\boldsymbol{\Omega}}) \mathrm{Z}(\mathbf{r}) \overline{\mathrm{E}}(\mathbf{r}) \mathrm{d}^{2} \widehat{\boldsymbol{\Omega}}$.

Inserting the expression of the total field $\bar{E}(\mathbf{r})$ as given by Eq. (17) in Eq. (28), and using the relation $\mathrm{d}^{3} \mathbf{r}^{\prime}=r^{\prime 2} \mathrm{~d} r^{\prime} \mathrm{d}^{2} \widehat{\boldsymbol{\Omega}}$, we are led to a Fredholm integral equation of the second kind for the radial amplitude vector:

$$
\begin{aligned}
\mathrm{f}_{m n}(r) & =\sum_{m^{\prime} n^{\prime}} \mathrm{U}_{m n, m^{\prime} n^{\prime}}(r) \mathrm{J}_{n^{\prime}}(r) \mathrm{a}_{m^{\prime} n^{\prime}} \\
& +\sum_{m^{\prime} n^{\prime}} \int_{0}^{R} \mathrm{U}_{m n, m^{\prime} n^{\prime}}(r) \mathrm{G}_{n^{\prime}}\left(r, r^{\prime}\right) \mathrm{f}_{m^{\prime} n^{\prime}}\left(r^{\prime}\right) \mathrm{d} r^{\prime} .
\end{aligned}
$$

In Eq. (29), the $3 \times 3$ matrix $U_{m n, m^{\prime} n^{\prime}}(r)$ is defined by

$\mathrm{U}_{m n, m^{\prime} n^{\prime}}(r)=r^{2} \int_{4 \pi} \chi(\mathbf{r}) \mathrm{Y}_{-m n}^{T}(\widehat{\boldsymbol{\Omega}}) \mathrm{Z}(\mathbf{r}) \mathrm{Y}_{m^{\prime} n^{\prime}}(\widehat{\boldsymbol{\Omega}}) \mathrm{d}^{2} \widehat{\boldsymbol{\Omega}}$.

Similarly, inserting Eq. (17) in Eq. (23) we find that the scattered field coefficients can be expressed in terms of $f_{m n}(r)$ as

$\mathrm{s}_{m n}=\frac{\mathrm{j} k_{0}}{\pi} \int_{0}^{R} \mathrm{~J}_{n}^{T}(r) \mathrm{f}_{m n}(r) \mathrm{d} r$.

In the following, we introduce the $3 \times 2$ matrix $F_{m n, m^{\prime} n^{\prime}}$ as the solution of the Fredholm integral equation of the second kind:

$$
\begin{aligned}
\mathrm{F}_{m n, m^{\prime} n^{\prime}}(r) & =\mathrm{U}_{m n, m^{\prime} n^{\prime}}(r) \mathrm{J}_{n^{\prime}}(r) \\
& +\sum_{m^{\prime \prime} n^{\prime \prime}} \int_{0}^{R} \mathrm{U}_{m n, m^{\prime \prime} n^{\prime \prime}}(r) \mathrm{G}_{n^{\prime \prime}}\left(r, r^{\prime}\right) \mathrm{F}_{m^{\prime \prime} n^{\prime \prime}, m^{\prime} n^{\prime}}\left(r^{\prime}\right) \mathrm{d} r^{\prime} .
\end{aligned}
$$

From Eqs. (29) and (32), it is apparent that $f_{m n}(r)$ and $F_{m n, m^{\prime} n^{\prime}}(r)$ solve two Fredholm integral equations with the same kernel but with different forcing (matrix) functions. If $\mathrm{W}_{m n, m^{\prime} n^{\prime}}(r)$ is the forcing function of the integral Eq. (32), i.e., $W_{m n, m^{\prime} n^{\prime}}(r)=$ $\mathrm{U}_{m n, m^{\prime} n^{\prime}}(r) \mathrm{J}_{n^{\prime}}(r)$, then $\mathrm{w}_{m n}(r)=\sum_{m^{\prime} n^{\prime}} \mathrm{W}_{m n, m^{\prime} n^{\prime}}(r) \mathrm{a}_{m^{\prime} n^{\prime}}$ is the forcing function of the integral Eq. (29). As a result, we have

$\mathrm{f}_{m n}(r)=\sum_{m^{\prime} n^{\prime}} \mathrm{F}_{m n, m^{\prime} n^{\prime}}(r) \mathrm{a}_{m^{\prime} n^{\prime}}$, and from Eq. (31), we get

$\mathrm{s}_{m n}=\frac{\mathrm{j} k_{0}}{\pi} \sum_{m^{\prime} n^{\prime}}\left[\int_{0}^{R} \mathrm{~J}_{n}^{T}(r) \mathrm{F}_{m n, m^{\prime} n^{\prime}}(r) \mathrm{d} r\right] \mathrm{a}_{m^{\prime} n^{\prime}}$.

Finally, by means of Eqs. (26) and (34), we find the following integral representation for the $\mathrm{T}$ matrix:

$\mathrm{T}_{m n, m^{\prime} n^{\prime}}=\frac{\mathrm{j} k_{0}}{\pi} \int_{0}^{R} \mathrm{~J}_{n}^{T}(r) \mathrm{F}_{m n, m^{\prime} n^{\prime}}(r) \mathrm{d} r$.

Thus, after solving the Fredholm integral Eq. (32) for $F_{m n, m^{\prime} n^{\prime}}(r)$, the matrix elements of the transition matrix $\mathrm{T}_{m n, m^{\prime} n^{\prime}}$ are computed with the help of Eq. (35).

In the case of an axisymmetric particle, we have $m_{\mathrm{r}}(\mathbf{r})=$ $m_{r}(r, \theta)$; hence putting

$\mathrm{Y}_{m n}(\widehat{\boldsymbol{\Omega}})=\mathrm{e}^{\mathrm{j} m \varphi} \mathrm{Y}_{m n}(\theta)$,

we obtain

$\mathrm{U}_{m n, m^{\prime} n^{\prime}}(r)=\delta_{m m^{\prime}} \mathrm{U}_{m n n^{\prime}}(r)$,

with

$$
\begin{aligned}
\mathrm{U}_{m n n^{\prime}}(r)= & 2 \pi r^{2} \int_{0}^{\pi} \chi(r, \theta) \mathrm{Y}_{-m n}^{T}(\theta) \mathrm{Z}(r, \theta) \mathrm{Y}_{m n^{\prime}}(\theta) \sin \theta \mathrm{d} \theta \\
= & \pi k_{0}^{2} r^{2} \frac{1}{\sqrt{n(n+1)}} \frac{1}{\sqrt{n^{\prime}\left(n^{\prime}+1\right)}} \int_{0}^{\pi}\left[\varepsilon_{\mathrm{r}}(r, \theta)-1\right] \sin \theta \mathrm{d} \theta \\
= & {\left[\begin{array}{c}
m^{2} \pi_{n}^{|m|}(\theta) \pi_{n^{\prime} \mid}^{|m|}(\theta)+\tau_{n}^{|m|}(\theta) \tau_{n^{\prime} \mid}^{|m|}(\theta) \\
\mathrm{j} m\left[\pi_{n}^{|m|}(\theta) \tau_{n^{\prime}}^{|m|}(\theta)+\tau_{n}^{|m|}(\theta) \pi_{n^{\prime}}^{|m|}(\theta)\right] \\
0 \\
-\mathrm{j} m\left[\pi_{n}^{|m|}(\theta) \tau_{n^{\prime}}^{|m|}(\theta)+\tau_{n}^{|m|}(\theta) \pi_{n^{\prime}}^{|m|}(\theta)\right] \\
m^{2} \pi_{n}^{|m|}(\theta) \pi_{n^{\prime}}^{|m|}(\theta)+\tau_{n}^{|m|}(\theta) \tau_{n^{\prime}}^{|m|}(\theta)
\end{array}\right.}
\end{aligned}
$$

$\left.\begin{array}{c}0 \\ 0 \\ \sqrt{n(n+1)} \sqrt{n^{\prime}\left(n^{\prime}+1\right)} P_{n}^{|m|}(\cos \theta) P_{n^{\prime}}^{|m|}(\cos \theta) / \varepsilon(r, \theta)\end{array}\right]$

and $\varepsilon_{\mathrm{r}}(r, \theta)=m_{\mathrm{r}}^{2}(r, \theta)$. Note that in Eq. (36), the difference between $\mathrm{Y}_{m n}(\widehat{\boldsymbol{\Omega}})$ and $\mathrm{Y}_{m n}(\theta)$ is given by their arguments. Consequently, we find

$\mathrm{F}_{m n, m^{\prime} n^{\prime}}(r)=\delta_{m m^{\prime}} \mathrm{F}_{m n n^{\prime}}(r)$

and

$\mathrm{T}_{m n, m^{\prime} n^{\prime}}=\delta_{m m^{\prime}} \mathrm{T}_{m n n^{\prime}}$

while for any azimuthal mode $m$, Eqs. (32) and (35) become

$\mathrm{F}_{m n n^{\prime}}(r)=\mathrm{U}_{m n n^{\prime}}(r) \mathrm{J}_{n^{\prime}}(r)+\sum_{n^{\prime \prime}} \int_{0}^{R} \mathrm{U}_{m n n^{\prime \prime}}(r) \mathrm{G}_{n^{\prime \prime}}\left(r, r^{\prime}\right) \mathrm{F}_{m n^{\prime \prime} n^{\prime}}\left(r^{\prime}\right) \mathrm{d} r^{\prime}$

and

$\mathrm{T}_{m n n^{\prime}}=\frac{\mathrm{j} k_{0}}{\pi} \int_{0}^{R} \mathrm{~J}_{n}^{T}(r) \mathrm{F}_{m n n^{\prime}}(r) \mathrm{d} r$,

respectively. Thus, the scattering problem decouples over the azimuthal mode $m$, and separate solutions for each $m$ can be obtained.

Further on, for $n, n^{\prime}=1, \ldots, N$, where $N$ is the maximum expansion order, we define, for the azimuthal mode $m$, the global dense matrices 
$\mathrm{U}_{m}:=\left[\mathrm{U}_{m n n^{\prime}}\right]=\left[\begin{array}{llll}\mathrm{U}_{m 11} & \mathrm{U}_{m 12} & \ldots & \mathrm{U}_{m 1 N} \\ \mathrm{U}_{m 21} & \mathrm{U}_{m 22} & \ldots & \mathrm{U}_{m 2 N} \\ & & & \\ \mathrm{U}_{m N 1} & \mathrm{U}_{m N 2} & \ldots & \mathrm{U}_{m N N}\end{array}\right]$,

$\mathrm{F}_{m}:=\left[\mathrm{F}_{m n n^{\prime}}\right]$, and $\mathrm{T}_{m}:=\left[\mathrm{T}_{m n n^{\prime}}\right]$, and the azimuthal-independent, global diagonal matrices

$\mathrm{J}:=\left[\mathrm{J}_{n} \delta_{n n^{\prime}}\right]=\left[\begin{array}{cccc}\mathrm{J}_{1} & 0 & \ldots & 0 \\ 0 & \mathrm{~J}_{2} & \ldots & 0 \\ & & & \\ 0 & 0 & \ldots & \mathrm{J}_{N}\end{array}\right]$,

$\mathrm{H}:=\left[\mathrm{H}_{n} \delta_{n n^{\prime}}\right]$, and $\mathrm{G}:=\left[\mathrm{G}_{n} \delta_{n n^{\prime}}\right]$. With these notations, we express Eqs. (42) and (43) as

$\mathrm{F}_{m}(r, R)=\mathrm{U}_{m}(r) \mathrm{J}(r)+\int_{0}^{R} \mathrm{U}_{m}(r) \mathrm{G}\left(r, r^{\prime}\right) \mathrm{F}_{m}\left(r^{\prime}, R\right) \mathrm{d} r^{\prime}$,

and

$\mathrm{T}_{m}(R)=\frac{\mathrm{j} k_{0}}{\pi} \int_{0}^{R} \mathrm{~J}^{T}(r) \mathrm{F}_{m}(r, R) \mathrm{d} r$,

respectively, and Eq. (19) as

$\mathrm{G}\left(r, r^{\prime}\right)=\frac{\mathrm{j} k_{0}}{\pi} \begin{cases}\mathrm{H}(r) \mathrm{J}^{T}\left(r^{\prime}\right), & r>r^{\prime} \\ \mathrm{J}(r) \mathrm{H}^{T}\left(r^{\prime}\right), & r<r^{\prime} .\end{cases}$

The (matrix) function $\mathrm{G}\left(r, r^{\prime}\right)$ is not defined for $r^{\prime}=r$, and at this point, the function has a jump discontinuity with the left and right limits

$\mathrm{G}\left(r, r_{-}\right)=\mathrm{H}(r) \mathrm{J}^{T}(r)$ and $\mathrm{G}\left(r, r_{+}\right)=\mathrm{J}(r) \mathrm{H}^{T}(r)$,

respectively.

\subsection{Invariant embedding procedure}

In the invariant embedding method, the length of the integration interval $R$ is regarded as a variable, and for this reason, in Eqs. (46) and (47), the dependency of $F_{m}$ and $T_{m}$ on $R$ is indicated explicitly. Referring to Fig. $1 \mathrm{~b}$, we note that $R$ varies between the initial radius $R_{0}$ and the radius of the smallest circumscribing sphere $R_{\mathrm{c}}$. Physically, for a given $R$, we are dealing with a "truncated" particle, i.e., a partial volume of the particle, created by the intersection between a sphere of radius $R$ and the particle. In this section we derive the central and forward recurrence relations for the $T$ matrix.

To derive the central recurrence relation, we consider the integral Eq. (46) in which we make the replacement $R \rightarrow R+\triangle R$ and set $r=R$; we obtain

$$
\begin{aligned}
\mathrm{F}_{m}(R, R+\Delta R) & =\mathrm{U}_{m}(R) \mathrm{J}(R) \\
& +\frac{\mathrm{j} k_{0}}{\pi} \mathrm{U}_{m}(R) \mathrm{H}(R) \int_{0}^{R-\Delta R} \mathrm{~J}^{T}(r) \mathrm{F}_{m}(r, R+\Delta R) \mathrm{d} r \\
& +\int_{R-\Delta R}^{R+\Delta R} \mathrm{U}_{m}(R) \mathrm{G}(R, r) \mathrm{F}_{m}(r, R+\Delta R) \mathrm{d} r .
\end{aligned}
$$

The last integral in Eq. (49) is of the form $\int_{R-\Delta R}^{R+\Delta R} f(r) \mathrm{d} r$, where $f(r)$ is a discontinuous function at $r=R$ with the left and right limits $f\left(R_{-}\right)$and $f\left(R_{+}\right)$, respectively. Representing this integral as the sum of two integrals, namely $\int_{R-\Delta R}^{R} f(r) \mathrm{d} r$ and $\int_{R}^{R+\Delta R} f(r) \mathrm{d} r$, we consider a quadrature method in which the first integral is computed by the right-endpoint quadrature formula $\int_{R-\triangle R}^{R} f(r) \mathrm{d} r=$ $\triangle R f\left(R_{-}\right)$, and the second integral is computed by the left-endpoint quadrature formula $\int_{R}^{R+\Delta R} f(r) \mathrm{d} r=\Delta R f\left(R_{+}\right)$. The result is a midpoint quadrature formula for the discontinuous function $f(r)$, that is, $\int_{R-\triangle R}^{R+\Delta R} f(r) \mathrm{d} r=2 \triangle R f(R)$, with $f(R)=\left[f\left(R_{-}\right)+f\left(R_{+}\right)\right] / 2$. Making use on this result, we obtain

$$
\begin{aligned}
\mathrm{F}_{m}(R, R+\Delta R) & =\mathrm{U}_{m}(R) \mathrm{J}(R) \\
& +\frac{\mathrm{j} k_{0}}{\pi} \mathrm{U}_{m}(R) \mathrm{H}(R) \int_{0}^{R-\Delta R} \mathrm{~J}^{T}(r) \mathrm{F}_{m}(r, R+\Delta R) \mathrm{d} r \\
& +2 \triangle R \mathrm{U}_{m}(R) \mathrm{G}(R, R) \mathrm{F}_{m}(R, R+\Delta R),
\end{aligned}
$$

where we have set

$\mathrm{G}(R, R)=\frac{1}{2}\left[\mathrm{G}\left(R, R_{-}\right)+\mathrm{G}\left(R, R_{+}\right)\right]$.

Using Eq. (50) as a starting point, we arrive at the central recurrence relation:

$$
\begin{aligned}
\mathrm{T}_{m}(R) & =\mathrm{Q}_{m}^{11}+\left(\mathrm{I}+\mathrm{Q}_{m}^{12}\right)\left[\mathrm{I}-\mathrm{T}_{m}(R-\Delta R) \mathrm{Q}_{m}^{22}\right]^{-1} \\
& \times \mathrm{T}_{m}(R-\Delta R)\left(\mathrm{I}+\mathrm{Q}_{m}^{21}\right),
\end{aligned}
$$

where

$\mathrm{Q}_{m}^{i j}=\mathrm{Q}_{m}^{i j}\left(R-\frac{\triangle R}{2}, \Delta R\right), \quad i, j=1,2$,

and in general,

$\mathrm{Q}_{m}^{11}(R, \triangle R)=\frac{\mathrm{j} k_{0}}{\pi} \mathrm{J}^{T}(R) \mathrm{V}_{m}(R, \triangle R) \mathrm{J}(R)$,

$\mathrm{Q}_{m}^{12}(R, \triangle R)=\frac{\mathrm{j} k_{0}}{\pi} \mathrm{J}^{T}(R) \mathrm{V}_{m}(R, \triangle R) \mathrm{H}(R)$,

$\mathrm{Q}_{m}^{21}(R, \Delta R)=\frac{\mathrm{j} k_{0}}{\pi} \mathrm{H}^{T}(R) \mathrm{V}_{m}(R, \triangle R) \mathrm{J}(R)$,

$\mathrm{Q}_{m}^{22}(R, \Delta R)=\frac{\mathrm{j} k_{0}}{\pi} \mathrm{H}^{T}(R) \mathrm{V}_{m}(R, \triangle R) \mathrm{H}(R)$,

with

$\mathrm{V}_{m}(R, \Delta R)=\triangle R\left[\mathrm{I}-\triangle R \mathrm{U}_{m}(R) \mathrm{G}(R, R)\right]^{-1} \mathrm{U}_{m}(R)$.

The derivation of the recurrence (52) is outlined in Appendix A.

To obtain the forward recurrence relation we consider again the integral equations (46) with $r=R$, that is,

$\mathrm{F}_{m}(R, R)=\mathrm{U}_{m}(R) \mathrm{J}(R)$

$$
\begin{aligned}
& +\frac{\mathrm{j} k_{0}}{\pi} \mathrm{U}_{m}(R) \mathrm{H}(R) \int_{0}^{R-\Delta R} \mathrm{~J}^{T}(r) \mathrm{F}_{m}(r, R) \mathrm{d} r \\
& +\int_{R-\Delta R}^{R} \mathrm{U}_{m}(R) \mathrm{G}(R, r) \mathrm{F}_{m}(r, R) \mathrm{d} r .
\end{aligned}
$$

The integrand of the last integral, denoted by $f(r)$, is not defined at $r=R$ but has the left limit $f\left(R_{-}\right)$. Computing this integral by means of the right-endpoint quadrature formula $\int_{R-\Delta R}^{R} f(r) \mathrm{d} r \approx$ $\triangle R f\left(R_{-}\right)$, we obtain

$$
\begin{aligned}
\mathrm{F}_{m}(R, R) & =\mathrm{U}_{m}(R) \mathrm{J}(R)+\frac{\mathrm{j} k_{0}}{\pi} \mathrm{U}_{m}(R) \mathrm{H}(R) \int_{0}^{R-\Delta R} \mathrm{~J}^{T}(r) \mathrm{F}_{m}(r, R) \mathrm{d} r \\
& +\triangle R \mathrm{U}_{m}(R) \mathrm{G}\left(R, R_{-}\right) \mathrm{F}_{m}(R, R) .
\end{aligned}
$$

Making the replacement $\mathrm{G}\left(R, R_{-}\right) \rightarrow \mathrm{G}(R, R)$, with $\mathrm{G}(R, R)$ as in Eq. (51), we are led to the same recurrence relation (52), but in which the matrices $Q_{m}^{i j}$ are now given by (compare with Eq. (53))

$\mathrm{Q}_{m}^{i j}=\mathrm{Q}_{m}^{i j}(R, \Delta R), \quad i, j=1,2$.

Thus, the difference between the two recurrence relations is that in the central scheme, the matrices $Q_{m}^{i j}$ are evaluated at the midpoint $R-\Delta R / 2$ of the interval $[R-\Delta R, R]$, while in the forward scheme, the matrices $Q_{m}^{i j}$ are evaluated at the right endpoint $R$ of the interval $[R-\triangle R, R]$.

In view of Eqs. (52) and (58), it is apparent that the recurrence (52) requires the inversion of two $2 N_{\max } \times 2 N_{\max }$ matrices, where $N_{\max }=N-|m|+1$. 


\subsection{The matrix Riccati equation}

The derivation of the matrix Riccati equation for the $\mathrm{T}$ matrix involves the following steps [27]:

1. Taking the derivative of Eq. (46) with respect to $R$, and using the relation $\mathrm{G}(r, R)=\left(\mathrm{j} k_{0} / \pi\right) \mathrm{J}(r) \mathrm{H}^{T}(R)$ for $r<R$, we find

$$
\begin{aligned}
\frac{\partial \mathrm{F}_{m}}{\partial R}(r, R) & =\frac{\mathrm{j} k_{0}}{\pi} \mathrm{U}_{m}(r) \mathrm{J}(r) \mathrm{H}^{T}(R) \mathrm{F}_{m}(R, R) \\
& +\int_{0}^{R} \mathrm{U}_{m}(r) \mathrm{G}\left(r, r^{\prime}\right) \frac{\partial \mathrm{F}_{m}}{\partial R}\left(r^{\prime}, R\right) \mathrm{d} r^{\prime} .
\end{aligned}
$$

The Fredholm integral equations (46) and (62) have the same kernel but their forcing functions differ by a multiplicative constant; hence, we deduce that

$$
\frac{\partial \mathrm{F}_{m}}{\partial R}(r, R)=\frac{\mathrm{j} k_{0}}{\pi} \mathrm{F}_{m}(r, R) \mathrm{H}^{T}(R) \mathrm{F}_{m}(R, R)
$$

for $r<R$.

2. Taking the derivative of Eq. (47) with respect to $R$, and substituting Eq. (63) in the integrand of the resulting equation, we obtain

$$
\frac{\mathrm{dT}_{m}}{\mathrm{~d} R}(R)=\frac{\mathrm{j} k_{0}}{\pi} \mathrm{J}^{T}(R) \mathrm{F}_{m}(R, R)+\frac{\mathrm{j} k_{0}}{\pi} \mathrm{T}_{m}(R) \mathrm{H}^{T}(R) \mathrm{F}_{m}(R, R) .
$$

3. Setting $r=R$ in Eq. (46), and using the relation $\mathrm{G}\left(R, r^{\prime}\right)=$ $\left(\mathrm{j} k_{0} / \pi\right) \mathrm{H}(R) \mathrm{J}^{T}\left(r^{\prime}\right)$ for $r^{\prime}<R$ along with Eq. (47), we find that $\mathrm{F}_{m}(R, R)$, which enters in Eq. (64), computes as

$$
\mathrm{F}_{m}(R, R)=\mathrm{U}_{m}(R) \mathrm{J}(R)+\mathrm{U}_{m}(R) \mathrm{H}(R) \mathrm{T}_{m}(R) .
$$

4. Inserting Eq. (65) in Eq. (64), we obtain the following matrix Riccati equation for the $T$ matrix:

$$
\begin{aligned}
\frac{\mathrm{dT}_{m}}{\mathrm{~d} R}(R) & =\overline{\mathrm{Q}}_{m}^{11}(R)+\overline{\mathrm{Q}}_{m}^{12}(R) \mathrm{T}_{m}(R) \\
& +\mathrm{T}_{m}(R) \overline{\mathrm{Q}}_{m}^{21}(R)+\mathrm{T}_{m}(R) \overline{\mathrm{Q}}_{m}^{22}(R) \mathrm{T}_{m}(R),
\end{aligned}
$$

where

$$
\begin{aligned}
& \overline{\mathrm{Q}}_{m}^{11}(R)=\frac{\mathrm{j} k_{0}}{\pi} \mathrm{J}^{T}(R) \mathrm{U}_{m}(R) \mathrm{J}(R), \\
& \overline{\mathrm{Q}}_{m}^{12}(R)=\frac{\mathrm{j} k_{0}}{\pi} \mathrm{J}^{T}(R) \mathrm{U}_{m}(R) \mathrm{H}(R), \\
& \overline{\mathrm{Q}}_{m}^{21}(R)=\frac{\mathrm{j} k_{0}}{\pi} \mathrm{H}^{T}(R) \mathrm{U}_{m}(R) \mathrm{J}(R), \\
& \overline{\mathrm{Q}}_{m}^{22}(R)=\frac{\mathrm{j} k_{0}}{\pi} \mathrm{H}^{T}(R) \mathrm{U}_{m}(R) \mathrm{H}(R) .
\end{aligned}
$$

The differential equation (66) is endowed with the initial condition $\mathrm{T}_{m}\left(R_{0}\right)$ and in particular, we have:

1. if $R_{0}=0, \mathrm{~T}_{m}\left(R_{0}\right)=0$,

2. if $R_{0}$ is the radius of a sphere enclosed in the particle, $\mathrm{T}_{m}\left(R_{0}\right)$ can be computed by the separation of variables method (Mie theory), and finally,

3. if $R_{0}$ is greater than the radius of the largest inscribed sphere, $\mathrm{T}_{m}\left(R_{0}\right)$ can be computed by the null-field method.

The numerical methods for solving the matrix Riccati equation are based on the transformation of the matrix quadratic equation into a system of linear, first-order differential equations by means of the Bernoulli substitution [28-31]. The theoretical basis for this substitution is the following result: If $\mathrm{U}(R)$ and $\mathrm{V}(R)$ solve the matrix differential equation $\frac{\mathrm{d}}{\mathrm{d} R}\left[\begin{array}{c}\mathrm{U}(R) \\ \mathrm{V}(R)\end{array}\right]=\mathrm{A}(R)\left[\begin{array}{c}\mathrm{U}(R) \\ \mathrm{V}(R)\end{array}\right]$,

with

$\mathrm{A}(R)=\left[\begin{array}{cc}-\overline{\mathrm{Q}}_{m}^{21}(R) & -\overline{\mathrm{Q}}_{m}^{22}(R) \\ \overline{\mathrm{Q}}_{m}^{11}(R) & \overline{\mathrm{Q}}_{m}^{12}(R)\end{array}\right]$,

then

$\mathrm{T}_{m}(R)=\mathrm{V}(R) \mathrm{U}^{-1}(R)$

solves the matrix Riccati equations (46).

With the goal of deriving recurrence relations for the transition matrix, we put

$\mathrm{X}(r)=\left[\begin{array}{c}\mathrm{U}(r) \\ \mathrm{V}(r)\end{array}\right]$,

and solve the matrix differential equation

$\frac{\mathrm{dX}}{\mathrm{d} r}(r)=\mathrm{A}(r) \mathrm{X}(r)$,

in the interval $[R-\triangle R, R]$ with the initial condition $\mathrm{X}(R-\triangle R)$. Assuming that in this interval, the matrix $\mathrm{A}(r)$ can be approximated by a constant matrix $\mathrm{A}_{0}(R)$, that is, $\mathrm{A}_{0}(R) \approx \mathrm{A}(r)$ for all $R-\Delta R \leq r \leq R$, we find that the solution of the differential equation (75) at $r=R$ is

$\mathrm{X}(R)=\mathrm{B}(R, \triangle R) \mathrm{X}(R-\triangle R)$,

where

$\mathrm{B}(R, \Delta R)=\mathrm{e}^{\Delta R \mathrm{~A}_{0}(R)}=\left[\begin{array}{ll}\mathrm{B}_{11}(R, \Delta R) & \mathrm{B}_{12}(R, \Delta R) \\ \mathrm{B}_{21}(R, \Delta R) & \mathrm{B}_{22}(R, \Delta R)\end{array}\right]$

is the fundamental matrix of the differential equation (75). From Eqs. (73), (74) and (76)-(77), we obtain the recurrence relation:

$$
\begin{aligned}
\mathrm{T}_{m}(R) & =\left[\mathrm{B}_{21}(R, \Delta R)+\mathrm{B}_{22}(R, \Delta R) \mathrm{T}_{m}(R-\Delta R)\right] \\
& \times\left[\mathrm{B}_{11}(R, \Delta R)+\mathrm{B}_{12}(R, \Delta R) \mathrm{T}_{m}(R-\Delta R)\right]^{-1} .
\end{aligned}
$$

Provided that the matrix $\mathrm{A}_{0}(R)$ is known, the matrix exponential in Eq. (77) can be computed by means of the Pade approximation. We note that the $n$th order Pade approximation is

$\mathrm{B}(R, \triangle R)=\left[\mathrm{D}_{n}\left(\triangle R \mathrm{~A}_{0}(R)\right)\right]^{-1} \mathrm{~N}_{n}\left(\triangle R \mathrm{~A}_{0}(R)\right)$,

$\mathrm{D}_{n}\left(\triangle R \mathrm{~A}_{0}(R)\right)=\sum_{k=0}^{n}(-1)^{k} c_{k}(\triangle R)^{k} \mathrm{~A}_{0}^{k}(R)$,

$\mathrm{N}_{n}\left(\triangle R \mathrm{~A}_{0}(R)\right)=\sum_{k=0}^{n} c_{k}(\triangle R)^{k} \mathrm{~A}_{0}^{k}(R)$,

where

$c_{k}=\frac{(2 n-k) ! n !}{(2 n) ! k !(n-k) !}$,

while the first-order approximation is

$\mathrm{B}(R, \triangle R)=\left[\mathrm{I}-\frac{\triangle R}{2} \mathrm{~A}_{0}(R)\right]^{-1}\left[\mathrm{I}+\frac{\triangle R}{2} \mathrm{~A}_{0}(R)\right]$.

Depending on the choice of the matrix $A_{0}(R)$ in the interval $[R-\triangle R, R]$ we obtain either a central or a forward recurrence relation. In the central scheme, the matrix $A(r)$, defined by Eq. (72), is approximated by its value at the midpoint $r=R-\triangle R / 2$ :

$\mathrm{A}_{0}(R) \approx \mathrm{A}\left(R-\frac{\triangle R}{2}\right)$,

while in the forward scheme, the matrix $A(r)$ is approximated by its value at the right endpoint $r=R$ :

$\mathrm{A}_{0}(R) \approx \mathrm{A}(R)$. 


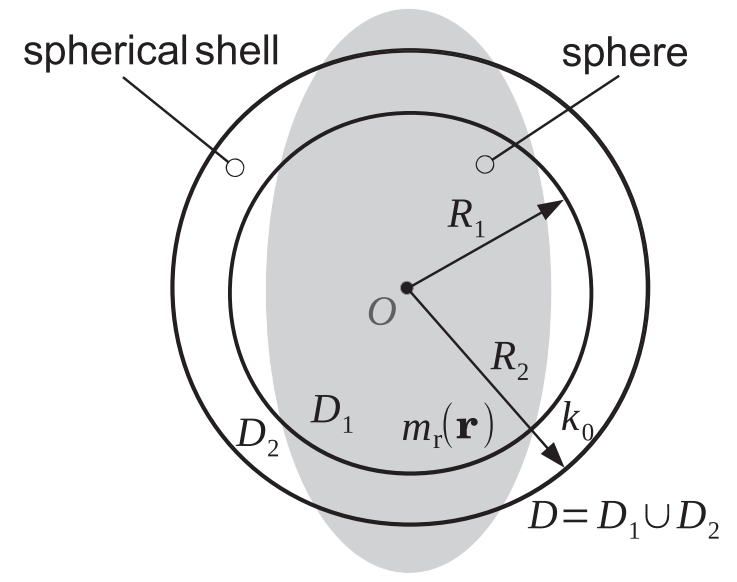

Fig. 2. Illustration of the superposition T-matrix method.

Thus, in the framework of the first-order Pade approximation, the recurrence relation is Eq. (78) in conjunction with Eq. (83); the difference between the central and the forward scheme consists in the approximations for $\mathrm{A}(r)$ given by Eqs. (84) and (85).

From Eqs. (78) and (83), we infer that the recurrence (78) requires the inversion of a $4 N_{\max } \times 4 N_{\max }$ matrix and of a $2 N_{\max } \times 2 N_{\max }$ matrix. However, as shown in Appendix B, the inversion of the $4 N_{\max } \times 4 N_{\max }$ matrix $\mathrm{B}=\mathrm{B}(R, \Delta R)$ is equivalent to the inversion of two $2 N_{\max } \times 2 N_{\max }$ matrices; hence, roughly speaking, the recurrence (78) requires the inversion of three $2 N_{\max } \times 2 N_{\max }$ matrices.

Note that the recurrence relations of Section 2.3 can be regarded as alternative algorithms for generating numerical solutions to the matrix Riccati Eq. (66). Also note that if the matrix exponential is computed by means of the first-order Taylor approximation

$\mathrm{e}^{\triangle R \mathrm{~A}_{0}(R)} \approx \mathrm{I}+\triangle R \mathrm{~A}_{0}(R)$

we are led to the recurrence relation

$$
\begin{aligned}
\mathrm{T}_{m}(R) & =\left[\left(\mathrm{I}+\Delta R \overline{\mathrm{Q}}_{m}^{12}\right) \mathrm{T}_{m}(R-\Delta R)+\triangle R \overline{\mathrm{Q}}_{m}^{11}\right] \\
& \times\left[\left(\mathrm{I}-\triangle R \overline{\mathrm{Q}}_{m}^{21}\right)-\Delta R \overline{\mathrm{Q}}_{m}^{22} \mathrm{~T}_{m}(R-\Delta R)\right]^{-1} .
\end{aligned}
$$

This recurrence, which demands only one inversion of a $2 N_{\max } \times 2 N_{\max }$ matrix, has been derived in Ref. [27]. However, as our numerical simulations have confirmed, the recurrence (87) has a low order of approximation, and so, it is less accurate than the recurrence (78). For this reason, it does not present any practical interest.

\section{The superposition T-matrix method}

In Ref. [27] we described a version of the superposition Tmatrix method, which enabled us to obtain a forward recurrence relation for the $T$ matrix, without invoking the invariant embedding procedure. In this section, we reiterate the proof by employing more rigorous arguments with the goal of introducing central and forward recurrence relations.

\subsection{The Fredholm integral equation for the radial amplitude vector}

Let us consider two concentric spheres of radii $R_{1}$ and $R_{2}>R_{1}$, enclosing an inhomogeneous region as shown in Fig. 2. The inhomogeneous sphere of radius $R_{2}$ is regarded as a system of two particles: the (inhomogeneous) sphere of radius $R_{1}$, and the (inhomogeneous) spherical shell between $R_{1}$ and $R_{2}$. The interior of the sphere of radius $R_{2}$ is denoted by $D$, the interior of the sphere of radius $R_{1}$ by $D_{1}$, and the domain corresponding to the spherical shell by $D_{2}$. As $D=\bar{D}_{1} \cup D_{2}$, the matrix-form representation of the volume integral equation (17) is

$$
\begin{aligned}
\overline{\mathrm{E}}(\mathbf{r}) & =\mathrm{E}_{0}(\mathbf{r})+\sum_{m n} \mathrm{Y}_{m n}(\widehat{\boldsymbol{\Omega}}) \int_{D_{1}} \chi\left(\mathbf{r}^{\prime}\right) \mathrm{G}_{n}\left(r, r^{\prime}\right) \mathrm{Y}_{-m n}^{T}\left(\widehat{\boldsymbol{\Omega}}^{\prime}\right) \mathrm{Z}\left(\mathbf{r}^{\prime}\right) \overline{\mathrm{E}}\left(\mathbf{r}^{\prime}\right) \mathrm{d}^{3} \mathbf{r}^{\prime} \\
& +\sum_{m n} \mathrm{Y}_{m n}(\widehat{\boldsymbol{\Omega}}) \int_{D_{2}} \chi\left(\mathbf{r}^{\prime}\right) \mathrm{G}_{n}\left(r, r^{\prime}\right) \mathrm{Y}_{-m n}^{T}\left(\widehat{\boldsymbol{\Omega}}^{\prime}\right) \mathrm{Z}\left(\mathbf{r}^{\prime}\right) \overline{\mathrm{E}}\left(\mathbf{r}^{\prime}\right) \mathrm{d}^{3} \mathbf{r}^{\prime}
\end{aligned}
$$

In the superposition T-matrix method, we distinguish the following fields.

1. The field scattered by the spherical shell in $D_{1}$ is $\left(\mathbf{r} \in D_{1}\right)$

$$
\begin{aligned}
\mathrm{E}_{\mathbf{s}}^{2}(\mathbf{r}) & =\sum_{m n} \mathrm{Y}_{m n}(\widehat{\boldsymbol{\Omega}}) \int_{D_{2}} \chi\left(\mathbf{r}^{\prime}\right) \mathrm{G}_{n}\left(r, r^{\prime}\right) \mathrm{Y}_{-m n}^{T}\left(\widehat{\boldsymbol{\Omega}}^{\prime}\right) \mathrm{Z}\left(\mathbf{r}^{\prime}\right) \overline{\mathrm{E}}\left(\mathbf{r}^{\prime}\right) \mathrm{d}^{3} \mathbf{r}^{\prime} \\
& =\sum_{m n} \mathbf{Y}_{m n}(\widehat{\boldsymbol{\Omega}}) \mathrm{J}_{n}(r) \mathbf{a}_{m n}^{2}
\end{aligned}
$$

where

$\mathrm{a}_{m n}^{2}=\frac{\mathrm{j} k_{0}}{\pi} \int_{R_{1}}^{R_{2}} \mathrm{H}_{n}^{T}(r) \mathrm{f}_{m n}(r) \mathrm{d} r$

are the expansion coefficients of $E_{s}^{2}(\mathbf{r})$ in terms of the regular functions $J_{n}(r)$. This field excites the sphere of radius $R_{1}$.

2. The field scattered by the sphere of radius $R_{1}$ in $D_{2}$ is $\left(\mathbf{r} \in D_{2}\right)$

$$
\begin{aligned}
\mathrm{E}_{\mathbf{s}}^{1}(\mathbf{r}) & =\sum_{m n} \mathbf{Y}_{m n}(\widehat{\boldsymbol{\Omega}}) \int_{D_{1}} \chi\left(\mathbf{r}^{\prime}\right) \mathrm{G}_{n}\left(r, r^{\prime}\right) \mathrm{Y}_{-m n}^{T}\left(\widehat{\boldsymbol{\Omega}}^{\prime}\right) \mathrm{Z}\left(\mathbf{r}^{\prime}\right) \overline{\mathrm{E}}\left(\mathbf{r}^{\prime}\right) \mathrm{d}^{3} \mathbf{r}^{\prime} \\
& =\sum_{m n} \mathbf{Y}_{m n}(\widehat{\boldsymbol{\Omega}}) \mathrm{H}_{n}(r) \mathbf{s}_{m n}^{1}
\end{aligned}
$$

where

$\mathrm{s}_{m n}^{1}=\frac{\mathrm{j} k_{0}}{\pi} \int_{0}^{R_{1}} \mathrm{~J}_{n}^{T}(r) \mathrm{f}_{m n}(r) \mathrm{d} r$

are the expansion coefficients of $E_{s}^{1}(\mathbf{r})$ in terms of the radiating functions $\mathrm{H}_{n}(r)$. This field excites the spherical shell.

3. The total field exciting the sphere of radius $R_{1}$ is

$\mathrm{E}_{\mathbf{s}}^{2}(\mathbf{r})+\mathrm{E}_{0}(\mathbf{r})=\sum_{m n} \mathrm{Y}_{m n}(\widehat{\boldsymbol{\Omega}}) \mathrm{J}_{n}(r)\left(\mathrm{a}_{m n}+\mathrm{a}_{m n}^{2}\right)$,

while the total field exciting the spherical shell is

$\mathrm{E}_{\mathbf{s}}^{1}(\mathbf{r})+\mathrm{E}_{0}(\mathbf{r})=\sum_{m n} \mathbf{Y}_{m n}(\widehat{\boldsymbol{\Omega}})\left[\mathrm{J}_{n}(r) \mathrm{a}_{m n}+\mathrm{H}_{n}(r) \mathbf{s}_{m n}^{1}\right]$.

Noting that the exciting field (93) possesses an expansion in terms of regular functions, we have the T-matrix equation

$s_{m n}^{1}=\sum_{m^{\prime} n^{\prime}} \mathrm{T}_{m n, m^{\prime} n^{\prime}}^{1}\left(\mathrm{a}_{m^{\prime} n^{\prime}}+\mathrm{a}_{m^{\prime} n^{\prime}}^{2}\right)$,

where $T_{m n, m^{\prime} n^{\prime}}^{1}$ is the transition matrix of the sphere of radius $R_{1}$.

4. The field scattered by the system of particles (the sphere of radius $R_{2}$ ) sums the contributions of the fields scattered by the sphere of radius $R_{1}$ and the spherical shell; it is given by $\left(\mathbf{r} \in \mathbb{R}^{3} \backslash \bar{D}\right)$

$$
\begin{aligned}
\mathbf{E}_{\mathbf{s}}(\mathbf{r}) & =\sum_{m n} \mathbf{Y}_{m n}(\widehat{\boldsymbol{\Omega}}) \mathrm{H}_{n}(r) \mathbf{s}_{m n}^{1} \\
& +\frac{\mathrm{j} k_{0}}{\pi} \sum_{m n} \mathbf{Y}_{m n}(\widehat{\boldsymbol{\Omega}}) \mathrm{H}_{n}(r) \int_{R_{1}}^{R_{2}} \mathrm{~J}_{n}^{T}\left(r^{\prime}\right) \mathrm{f}_{m n}\left(r^{\prime}\right) \mathrm{d} r^{\prime} \\
& =\sum_{m n} \mathbf{Y}_{m n}(\widehat{\boldsymbol{\Omega}}) \mathrm{H}_{n}(r) \mathbf{s}_{m n},
\end{aligned}
$$

where 
$\mathrm{s}_{m n}=\mathrm{s}_{m n}^{1}+\frac{\mathrm{j} k_{0}}{\pi} \int_{R_{1}}^{R_{2}} \mathrm{~J}_{n}^{T}(r) \mathrm{f}_{m n}(r) \mathrm{d} r$

are the expansion coefficients of $E_{s}(\mathbf{r})$ in terms of the radiating functions $\mathrm{H}_{n}(r)$. For the sphere of radius $R_{2}$ characterized by the transition matrix $\mathrm{T}_{m n, m^{\prime} n^{\prime}}$, the T-matrix equation is

$\mathrm{s}_{m n}=\sum_{m^{\prime} n^{\prime}} \mathrm{T}_{m n, m^{\prime} n^{\prime}} \mathbf{a}_{m^{\prime} n^{\prime}}$

By means of Eq. (91), we express the volume integral Eq. (88) in $D_{2}$ as $\left(\mathbf{r} \in D_{2}\right)$

$$
\begin{aligned}
\overline{\mathrm{E}}(\mathbf{r}) & =\sum_{m n} \mathrm{Y}_{m n}(\widehat{\boldsymbol{\Omega}})\left[J_{n}(r) \mathrm{a}_{m n}+\mathrm{H}_{n}(r) \mathrm{s}_{m n}^{1}\right] \\
& +\sum_{m n} \mathbf{Y}_{m n}(\widehat{\boldsymbol{\Omega}}) \int_{D_{2}} \chi\left(\mathbf{r}^{\prime}\right) \mathrm{G}_{n}\left(r, r^{\prime}\right) \mathbf{Y}_{-m n}^{T}\left(\widehat{\boldsymbol{\Omega}}^{\prime}\right) \mathrm{Z}\left(\mathbf{r}^{\prime}\right) \overline{\mathrm{E}}\left(\mathbf{r}^{\prime}\right) \mathrm{d}^{3} \mathbf{r}^{\prime} .
\end{aligned}
$$

In view of Eq. (94), it is apparent that the first sum in the righthand side of Eq. (99) is the total field exciting the spherical shell. From Eq. (99) it follows that the radial amplitude vector, defined by Eq. (28), satisfies the integral equation

$$
\begin{aligned}
\mathrm{f}_{m n}(r) & =\sum_{m^{\prime} n^{\prime}} \mathrm{U}_{m n, m^{\prime} n^{\prime}}(r)\left[\mathrm{J}_{n^{\prime}}(r) \mathrm{a}_{m^{\prime} n^{\prime}}+\mathrm{H}_{n^{\prime}}(r) \mathrm{s}_{m^{\prime} n^{\prime}}^{1}\right] \\
& +\sum_{m^{\prime} n^{\prime}} \int_{R_{1}}^{R_{2}} \mathrm{U}_{m n, m^{\prime} n^{\prime}}(r) \mathrm{G}_{n^{\prime}}\left(r, r^{\prime}\right) \mathrm{f}_{m^{\prime} n^{\prime}}\left(r^{\prime}\right) \mathrm{d} r^{\prime},
\end{aligned}
$$

for $R_{1} \leq r \leq R_{2}$.

For an axisymmetric particle and in terms of global matrices, Eqs. (90), (95), (97), and (100) read as

$\mathrm{a}_{m}^{2}=\frac{\mathrm{j} k_{0}}{\pi} \int_{R_{1}}^{R_{2}} \mathrm{H}^{T}(r) \mathrm{f}_{m}(r) \mathrm{d} r$

$\mathrm{s}_{m}^{1}=\mathrm{T}_{m}^{1}\left(\mathrm{a}_{m}+\mathrm{a}_{m}^{2}\right)$,

$\mathrm{s}_{m}=\mathrm{s}_{m}^{1}+\frac{\mathrm{j} k_{0}}{\pi} \int_{R_{1}}^{R_{2}} \mathrm{~J}^{T}(r) \mathrm{f}_{m}(r) \mathrm{d} r$,

and

$\mathrm{f}_{m}(r)=\mathrm{U}_{m}(r)\left[\mathrm{J}(r) \mathrm{a}_{m}+\mathrm{H}(r) \mathrm{s}_{m}^{1}\right]+\int_{R_{1}}^{R_{2}} \mathrm{U}_{m}(r) \mathrm{G}\left(r, r^{\prime}\right) \mathrm{f}_{m}\left(r^{\prime}\right) \mathrm{d} r^{\prime}$,

respectively, while the T-matrix equation (98) takes the form $\mathrm{s}_{m}=$ $\mathrm{T}_{m} \mathrm{a}_{m}$. Here, we introduced the vector $\mathrm{f}_{m}:=\left[\mathrm{f}_{m n}\right]$ and in an analogous manner, the vectors $a_{m}, a_{m}^{2}, s_{m}$, and $s_{m}^{1}$.

\subsection{Recurrence relations for the transition matrix}

As in Section 2.3, central and forward recurrence relations for the transition matrix can be derived in the framework of the superposition T-matrix method.

We begin with the central recurrence relation. In Eq. (104) we choose $R_{1}=R-\triangle R$ and $R_{2}=R+\triangle R$, and set $r=R$. Using the midpoint quadrature formula for a discontinuous function, we find

$\mathrm{f}_{m}(R)=\mathrm{U}_{m}(R)\left[\mathrm{J}(R) \mathrm{a}_{m}+\mathrm{H}(R) \mathrm{s}_{m}^{1}\right]+2 \triangle R \mathrm{U}_{m}(R) \mathrm{G}(R, R) \mathrm{f}_{m}(R)$,

where $\mathrm{G}(R, R)$ is given by Eq. (51). We obtain the central recurrence relation

$$
\begin{aligned}
\mathrm{T}_{m}(R) & =\mathrm{T}_{m}(R-\Delta R)+\frac{\mathrm{j} k_{0}}{\pi} \Delta R\left[\mathrm{~T}_{m}(R-\Delta R) \mathrm{H}^{T}+\mathrm{J}^{T}\right] \\
& \times\left[\mathrm{I}-\triangle R \mathrm{U}_{m} \mathrm{G}-\frac{\mathrm{j} k_{0}}{\pi} \Delta R \mathrm{U}_{m} \mathrm{HT}_{m}(R-\triangle R) \mathrm{H}^{T}\right]^{-1}
\end{aligned}
$$

$$
\times \mathrm{U}_{m}\left[\mathrm{~J}+\mathrm{HT}_{m}(R-\triangle R)\right],
$$

where

$\mathrm{J}=\mathrm{J}\left(R-\frac{\triangle R}{2}\right), \quad \mathrm{H}=\mathrm{H}\left(R-\frac{\triangle R}{2}\right), \quad \mathrm{U}_{m}=\mathrm{U}_{m}\left(R-\frac{\triangle R}{2}\right)$

and

$\mathrm{G}=\mathrm{G}\left(R-\frac{\triangle R}{2}, R-\frac{\triangle R}{2}\right)$.

The derivation of the recurrence (106) is given in Appendix C.

To obtain the forward recurrence relation we consider Eq. (104) with $R_{1}=R-\triangle R$ and $R_{2}=R$, and set $r=R$. Using the right-endpoint quadrature formula, we obtain

$\mathrm{f}_{m}(R)=\mathrm{U}_{m}(R)\left[\mathrm{J}(R) \mathrm{a}_{m}+\mathrm{H}(R) \mathrm{s}_{m}^{1}\right]+\Delta R \mathrm{U}_{m}(R) \mathrm{G}\left(R, R_{-}\right) \mathrm{f}_{m}(R)$,

so that after the replacement $\mathrm{G}\left(R, R_{-}\right) \rightarrow \mathrm{G}(R, R)$, we are led to the forward recurrence relation (106) with

$\mathrm{J}=\mathrm{J}(R), \quad \mathrm{H}=\mathrm{H}(R), \quad \mathrm{U}_{m}=\mathrm{U}_{m}(R)$,

and

$\mathrm{G}=\mathrm{G}(R, R)$.

It is not difficult to see that the recurrence (106) demands the inversion of a $3 N_{\max } \times 3 N_{\max }$ matrix.

In terms of the matrix $V_{m}$ defined by Eq. (58), the recurrence relation (106) becomes

$$
\begin{aligned}
\mathrm{T}_{m}(R) & =\mathrm{T}_{m}(R-\triangle R)+\frac{\mathrm{j} k_{0}}{\pi}\left[\mathrm{T}_{m}(R-\triangle R) \mathrm{H}^{T}+\mathrm{J}^{T}\right] \\
& \times\left[\mathrm{I}-\frac{\mathrm{j} k_{0}}{\pi} \mathrm{V}_{m} \mathrm{HT}_{m}(R-\triangle R) \mathrm{H}^{T}\right]^{-1} \\
& \times \mathrm{V}_{m}\left[\mathrm{~J}+\mathrm{HT}_{m}(R-\triangle R)\right] .
\end{aligned}
$$

In Appendix D we show that the recurrences (52) and (112) are identical, which in turn implies, that the recurrences (52) and (106) are identical.

\section{Numerical simulations}

The recurrence schemes for T-matrix calculation have been implemented in a Fortran computer program. As in Refs. [24-26], the effective dimension of the $\mathrm{T}$ matrix increases with the radius $R$, and the Gaussian quadrature is employed for computing $U_{m}$. To improve the solution accuracy, the adaptive step-size procedure described in Appendix E has been implemented.

In this section we perform some simple numerical simulations in order to assess the accuracies and efficiencies of the central and forward recurrence schemes corresponding to the invariant embedding T-matrix method, the matrix Riccati equation method, and the superposition T-matrix method.

The particle considered in our analysis is a prolate spheroid with $k_{0} a=40$ and $k_{0} b=20$, where $a$ and $b$ are the polar (vertical) and the equatorial (horizontal) radius, respectively. The relative refractive index is $m_{r}=1.311$, and the initial spherical radius is $k_{0} R_{0}=30$. For simplicity, we consider that the incident direction is aligned with the symmetry axis of the spheroid. Moreover, for the simulations reported here, the adaptive step-size procedure is not used. The initial matrix $\mathrm{T}_{m}\left(R_{0}\right)$ is computed by using the single spherical coordinate-based null-field method. All simulations are performed with double-precision floating-point variables on a server Intel(R) Xeon(R) CPU E5-2695 v3 @ $2.30 \mathrm{GHz}$

We begin with a convergence test of the extinction efficiency $Q_{\text {ext }}$ over the expansion order $N$. The results are shown in Fig. 3. It is well known that the null-field method is characterized by a "semi-convergent" behavior: $Q_{\text {ext }}$ stagnates in a region called 

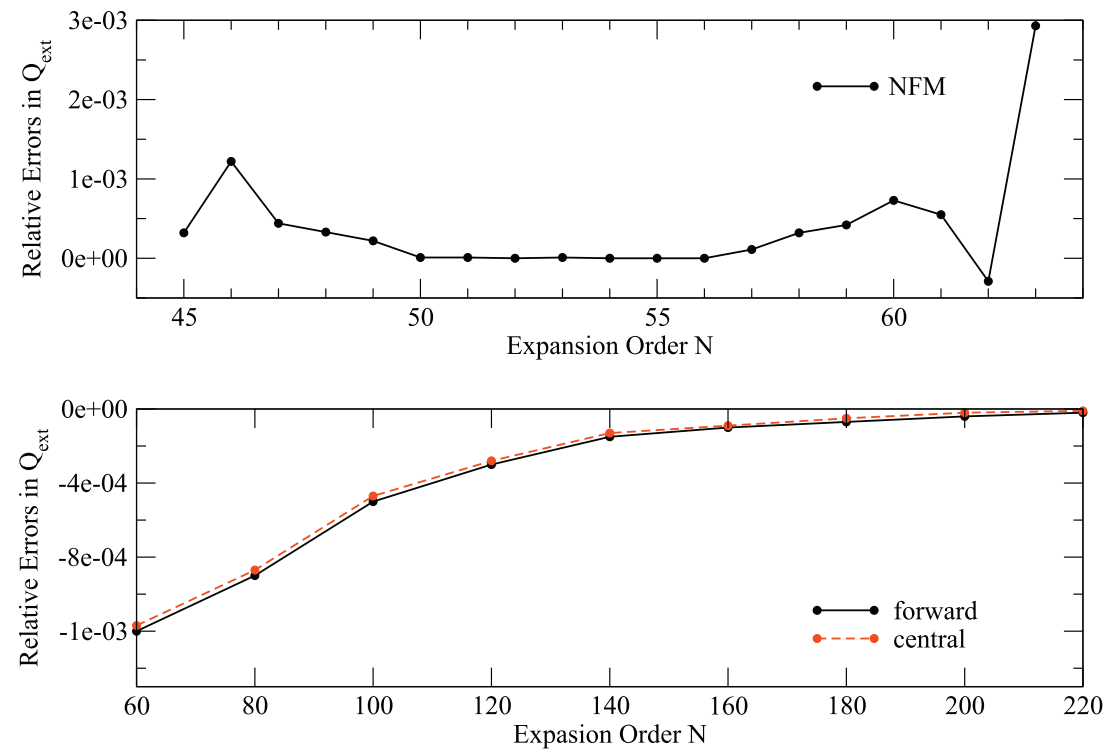

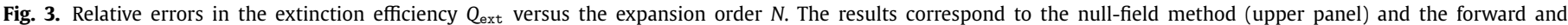
central recurrence schemes (lower panel). The step size is $k_{0} \triangle R=0.05$.

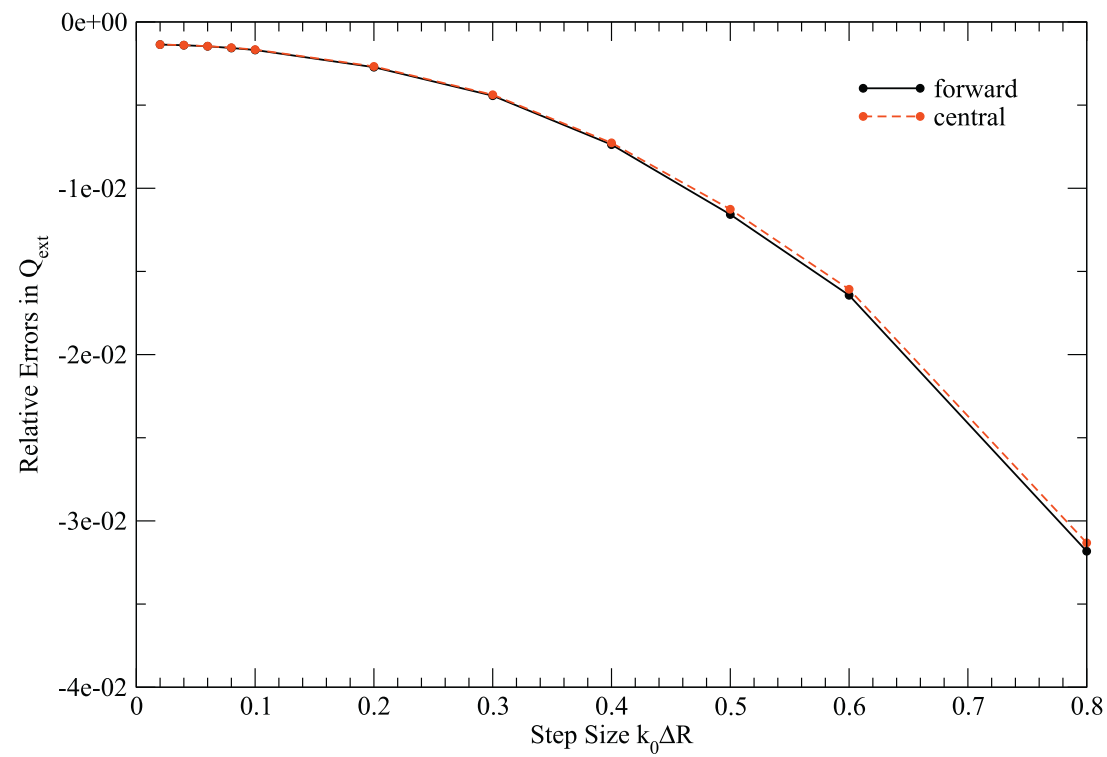

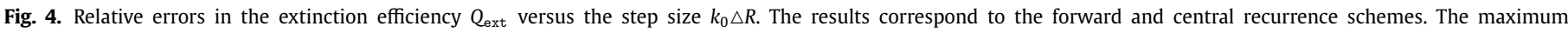
expansion order is $N=60$.

plateau of convergence, and increase or decrease outside this region. In the convergence region, delimited by $N=50$ and $N=56$, we found that $Q_{\text {ext0 }}=0.7883$ (upper panel of Fig. 3). In the lower panel of Fig. 3 we illustrate the relative errors in $Q_{\text {ext }}$ versus the expansion order $N$, taking $Q_{\text {ext0 }}$ as a reference. As expected, the plots show a "semi-convergent" behavior of the null-field method, and a monotonic convergent behavior of the recurrence schemes. What is impressive here is that these methods do not fail for large values of $N$. In fact, the results in Fig. 3 certify that Fredholm integral equations of the second kind are much less ill-posed than Fredholm integral equations of the first kind (they are not particularly ill-posed). In Fig. 4 we plot the relative errors in $Q_{\text {ext }}$ versus the step size $k_{0} \triangle R$. The results show that relative errors become smaller than $2 \cdot 10^{-3}$ for $k_{0} \Delta R \leq 0.1$. In addition to these conclusions we mention the following findings:
1. the computational result of all central recurrence schemes, as well as all forward recurrence schemes, are numerically identical (up to five digits);

2. the central recurrence schemes have a slightly better accuracy than the forward recurrence schemes (see lower panel of Figs. 3 and 4);

3. the convergence rates of the recurrence schemes is rather low.

In Fig. 5 we again plot the relative errors of $Q_{\text {ext }}$ versus the expansion order $N$, but now, we consider the extreme situation when the expansion order $N$ is very large. What we found is that the recurrence schemes of the superposition T-matrix method works up to $N=360$, while the recurrence schemes of the invariant embedding T-matrix method works only up to $N=220$; after this critical value, overflow errors occur. To extend the domain of applicabil- 


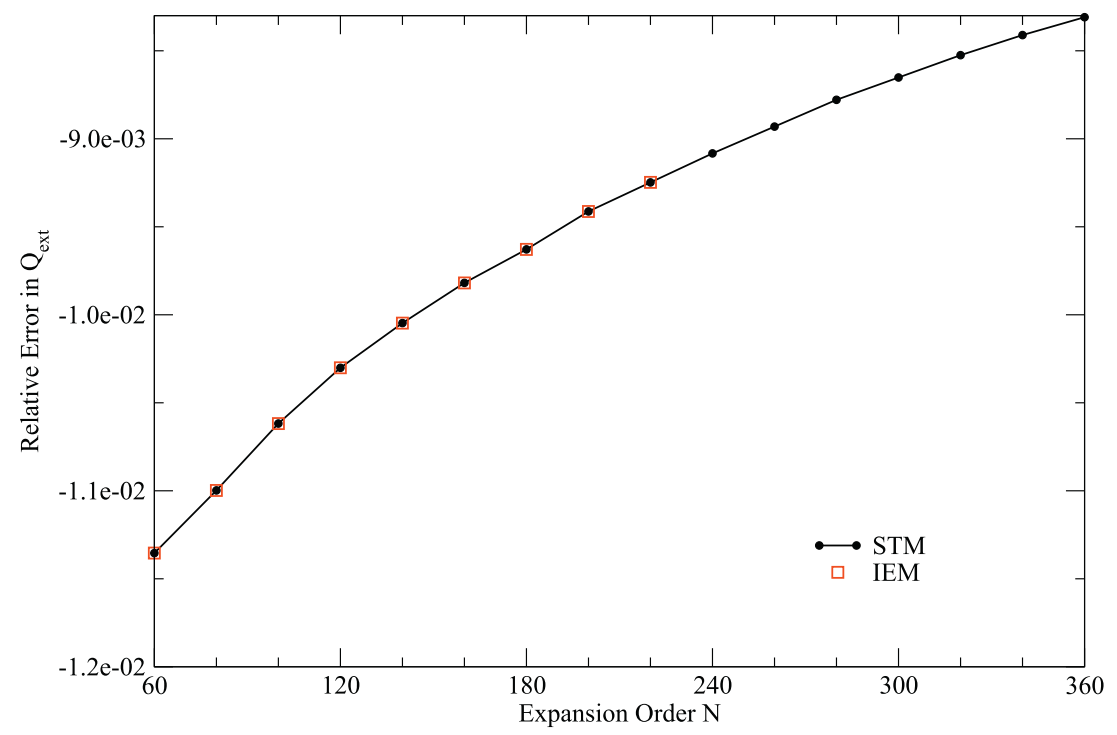

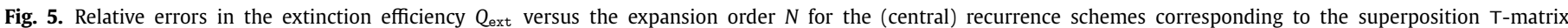
method (STM) and the invariant embedding T-matrix method (IEM). The step size is $k_{0} \triangle R=0.5$.

Table 1

Computation times in min:sec for the central recurrence schemes corresponding to the invariant embedding T-matrix method (IEM), the superposition Tmatrix method (STM), and the matrix Riccati equation method (MRM). The maximum expansion order increases from $N=60$ to $N=220$ in steps of 20 .

\begin{tabular}{llll}
\hline Step Size & IEM & STM & MRM \\
\hline 0.5 & $3: 39$ & $3: 38$ & $3: 56$ \\
0.25 & $7: 14$ & $6: 54$ & $7: 44$ \\
0.05 & $33: 38$ & $33: 04$ & $37: 31$ \\
\hline
\end{tabular}

ity of the latter method, we implemented a scaling procedure for computing the spherical Bessel and Neumann functions. This approach is described in Appendix F. With this scaling procedure, the recurrence schemes of the invariant embedding T-matrix method can be used up to $N=360$. Note that the recurrence schemes of the matrix Riccati equation method should be also equipped with this scaling procedure in order to achieve such performance. Also note that for $N>360$, all methods fail.

The computation times for the central recurrence schemes is shown in Table 1 . We recall, that the invariant embedding $\mathrm{T}$ matrix method requires the inversion of two $2 N_{\max } \times 2 N_{\max }$ matrices, the superposition T-matrix method of a $3 N_{\max } \times 3 N_{\max }$ matrix, and the matrix Riccati equation method of three $2 N_{\max } \times 2 N_{\max }$ matrices. The results show that the efficiencies of the recurrence schemes corresponding to the invariant embedding $T$-matrix method and the superposition T-matrix method are comparable, while the recurrence scheme corresponding to the matrix Riccati equation method is less efficient (but not dramatically less efficient).

\section{Conclusions}

In this paper, we reviewed several methods for deriving recurrence relations for T-matrix calculation. In particular, by performing a consistent analysis, we (i) derived central and forward recurrence relations in the framework of the invariant embedding Tmatrix method, the matrix Riccati equation method, and the superposition T-matrix method, and (ii) proved that the recurrence relations of the invariant embedding T-matrix method and the superposition T-matrix method are analytically equivalent. Although our paper is merely theoretical, we performed some simple computer simulations and found that

1. all central, as well as all forward recurrence schemes, are (almost) numerically identical,

2. the central recurrence schemes provide a slightly better accuracy than the forward recurrence schemes,

3. the recurrence schemes of the superposition T-matrix method are more stable than that of the invariant embedding T-matrix method and the matrix Riccati equation method,

4. the stability of the recurrence schemes corresponding to the invariant embedding T-matrix method and the matrix Riccati equation method can be increased by means of a scaling procedure for computing the spherical Bessel and Neumann functions,

5. the efficiencies of the recurrence schemes corresponding to the invariant embedding T-matrix method and the superposition T-matrix method are comparable.

It will be an interesting task for the scientists who developed efficient computer codes based on the forward recurrence scheme of the invariant embedding T-matrix method to implement these recurrence schemes in their codes, and to test them in complex applications (as reported in Refs. [24-26]).

\section{Appendix A}

In this appendix we derive the central and forward recurrence relations given by Eq. (52) in conjunction with Eqs. (53) and (61).

Central recurrence relation. The derivation involves the following steps.

Step 1. The integral equation (50), with $\mathrm{G}(R, R)$ given by Eq. (51), yields

$\mathrm{F}_{m}(R, R+\triangle R)=\frac{1}{2 \triangle R} \mathrm{~V}_{m}(R, 2 \triangle R)\left[\mathrm{J}(R)+\mathrm{H}(R) \mathrm{K}_{m}\right]$,

where the matrix $V_{m}$ is defined by Eq. (58), and the auxiliary matrix $\mathrm{K}_{m}$ is given by

$\mathrm{K}_{m}=\frac{\mathrm{j} k_{0}}{\pi} \int_{0}^{R-\Delta R} \mathrm{~J}^{T}(r) \mathrm{F}_{m}(r, R+\Delta R) \mathrm{d} r$ 
Step 2. Substituting Eq. (114) in the integral representation (47) for the T matrix with $R \rightarrow R+\triangle R$, gives

$\mathrm{T}_{m}(R+\Delta R)=\mathrm{K}_{m}+\frac{\mathrm{j} k_{0}}{\pi} \int_{R-\Delta R}^{R+\Delta R} \mathrm{~J}^{T}(r) \mathrm{F}_{m}(r, R+\Delta R) \mathrm{d} r$.

Computing the above integral by the midpoint quadrature formula $\int_{R-\Delta R}^{R+\Delta R} f(r) \mathrm{d} r=2 \Delta R f(R)$ (note that the integrand is a continuous function) and using Eq. (113), we obtain

$\mathrm{T}_{m}(R+\triangle R)=\mathrm{Q}_{m}^{11}(R, 2 \triangle R)+\left[\mathrm{I}+\mathrm{Q}_{m}^{12}(R, 2 \triangle R)\right] \mathrm{K}_{m}$,

where the matrices $Q_{m}^{i j}$ are given by Eqs. (54)-(57). is,

Step 3. Consider the integral Eq. (46) with $R \rightarrow R+\triangle R$, that

$$
\begin{aligned}
\mathrm{F}_{m}(r, R+\Delta R) & =\mathrm{U}_{m}(r) \mathrm{J}(r) \\
& +\int_{0}^{R-\Delta R} \mathrm{U}_{m}(r) \mathrm{G}\left(r, r^{\prime}\right) \mathrm{F}_{m}\left(r^{\prime}, R+\Delta R\right) \mathrm{d} r^{\prime} \\
& +\int_{R-\Delta R}^{R+\Delta R} \mathrm{U}_{m}(r) \mathrm{G}\left(r, r^{\prime}\right) \mathrm{F}_{m}\left(r^{\prime}, R+\Delta R\right) \mathrm{d} r^{\prime} .
\end{aligned}
$$

For $r \leq R-\triangle R$, application of the midpoint quadrature formula to the last integral yields

$$
\begin{aligned}
\mathrm{F}_{m}(r, R+\triangle R) & =\mathrm{U}_{m}(r) \mathrm{J}(r)\left[\mathrm{I}+2 \triangle R \frac{\mathrm{j} k_{0}}{\pi} \mathrm{H}^{T}(R) \mathrm{F}_{m}(R, R+\triangle R)\right] \\
& +\int_{0}^{R-\Delta R} \mathrm{U}_{m}(r) \mathrm{G}\left(r, r^{\prime}\right) \mathrm{F}_{m}\left(r^{\prime}, R+\Delta R\right) \mathrm{d} r^{\prime} .
\end{aligned}
$$

Next, consider the integral Eq. (46) with $R \rightarrow R-\triangle R$, that is,

$\mathrm{F}_{m}(r, R-\Delta R)=\mathrm{U}_{m}(r) \mathrm{J}(r)+\int_{0}^{R-\Delta R} \mathrm{U}_{m}(r) \mathrm{G}\left(r, r^{\prime}\right) \mathrm{F}_{m}\left(r^{\prime}, R-\Delta R\right) \mathrm{d} r^{\prime}$,

for $r \leq R-\triangle R$. Because for $r \leq R-\triangle R, \mathrm{~F}_{m}(r, R+\triangle R)$ and $\mathrm{F}_{m}(r, R-$ $\triangle R$ ) solve the same Fredholm integral equation but with forcing functions which differ by a multiplicative constant, we find that

$\mathrm{F}_{m}(r, R+\triangle R)=\mathrm{F}_{m}(r, R-\Delta R)\left(\mathrm{I}+\mathrm{L}_{m}\right)$,

where we have introduced the auxiliary matrix $L_{m}$ by the relation

$\mathrm{L}_{m}=2 \triangle R \frac{\mathrm{j} k_{0}}{\pi} \mathrm{H}^{T}(R) \mathrm{F}_{m}(R, R+\triangle R)$.

By means of Eq. (113), the matrix $L_{m}$ computes as

$\mathrm{L}_{m}=\mathrm{Q}_{m}^{21}(R, 2 \triangle R)+\mathrm{Q}_{m}^{22}(R, 2 \triangle R) \mathrm{K}_{m}$.

Step 4. The integral representation (47) for the $T$ matrix with $R \rightarrow R-\triangle R$ is

$\mathrm{T}_{m}(R-\Delta R)=\frac{\mathrm{j} k_{0}}{\pi} \int_{0}^{R-\Delta R} \mathrm{~J}^{T}(r) \mathrm{F}_{m}(r, R-\Delta R) \mathrm{d} r$.

Multiplying this equation from the right by $\mathrm{I}+\mathrm{L}_{m}$, and taking account of Eqs. (114) and (120), we get

$\mathrm{T}_{m}(R-\triangle R)\left(\mathrm{I}+\mathrm{L}_{m}\right)=\mathrm{K}_{m}$.

Step 5. Solving Eqs. (122) and (124) with respect to $\mathrm{K}_{m}$, we obtain

$$
\begin{aligned}
\mathrm{K}_{m} & =\left[\mathrm{I}-\mathrm{T}_{m}(R-\triangle R) \mathrm{Q}_{m}^{22}(R, 2 \triangle R)\right]^{-1} \\
& \times \mathrm{T}_{m}(R-\triangle R)\left[\mathrm{I}+\mathrm{Q}_{m}^{21}(R, 2 \triangle R)\right],
\end{aligned}
$$

so that by substituting this result in Eq. (116), we find

$$
\begin{aligned}
\mathrm{T}_{m}(R+\Delta R) & =\mathrm{Q}_{m}^{11}(R, 2 \triangle R)+\left[\mathrm{I}+\mathrm{Q}_{m}^{12}(R, 2 \triangle R)\right] \\
& \times\left[\mathrm{I}-\mathrm{T}_{m}(R-\triangle R) \mathrm{Q}_{m}^{22}(R, 2 \triangle R)\right]^{-1} \\
& \times \mathrm{T}_{m}(R-\triangle R)\left[\mathrm{I}+\mathrm{Q}_{m}^{21}(R, 2 \triangle R)\right] .
\end{aligned}
$$

Step 6. In Eq. (126) we make the successive transformations:

$\triangle R \rightarrow \frac{\triangle R}{2}$ and $R \rightarrow R-\frac{\triangle R}{2}$,

and obtain the central recurrence relation

$$
\begin{aligned}
\mathrm{T}_{m}(R) & =\mathrm{Q}_{m}^{11}\left(R-\frac{\triangle R}{2}, \Delta R\right)+\left[\mathrm{I}+\mathrm{Q}_{m}^{12}\left(R-\frac{\Delta R}{2}, \Delta R\right)\right] \\
& \times\left[\mathrm{I}-\mathrm{T}_{m}(R-\triangle R) \mathrm{Q}_{m}^{22}\left(R-\frac{\triangle R}{2}, \Delta R\right)\right]^{-1} \\
& \times \mathrm{T}_{m}(R-\triangle R)\left[\mathrm{I}+\mathrm{Q}_{m}^{21}\left(R-\frac{\triangle R}{2}, \Delta R\right)\right] .
\end{aligned}
$$

Forward recurrence relation. The derivation is similar to the previous one.

In Step 1, the integral equation (60) with $\mathrm{G}\left(R, R_{-}\right) \rightarrow \mathrm{G}(R, R)$ implies (compare with Eq. (113))

$\mathrm{F}_{m}(R, R)=\frac{1}{\triangle R} \mathrm{~V}_{m}(R, \Delta R)\left[\mathrm{J}(R)+\mathrm{H}(R) \mathrm{K}_{m}\right]$,

where $\mathrm{V}_{m}$ is as in Eq. (58), and

$\mathrm{K}_{m}=\frac{\mathrm{j} k_{0}}{\pi} \int_{0}^{R-\Delta R} \mathrm{~J}^{T}(r) \mathrm{F}_{m}(r, R) \mathrm{d} r$.

In Step 2, we consider the integral representation (47) for the T matrix

$\mathrm{T}_{m}(R)=\mathrm{K}_{m}+\frac{\mathrm{j} k_{0}}{\pi} \int_{R-\Delta R}^{R} \mathrm{~J}^{T}(r) \mathrm{F}_{m}(r, R) \mathrm{d} r$,

use the right-endpoint quadrature formula for a function which is continuous in the interval $[R-\triangle R, R]$, and find (compare with Eq. (116))

$\mathrm{T}_{m}(R)=\mathrm{Q}_{m}^{11}(R, \Delta R)+\left[\mathrm{I}+\mathrm{Q}_{m}^{12}(R, \Delta R)\right] \mathrm{K}_{m}$.

In Step 3, we derive the relationship (compare with Eq. (120))

$\mathrm{F}_{m}(r, R)=\mathrm{F}_{m}(r, R-\triangle R)\left(\mathrm{I}+\mathrm{L}_{m}\right)$,

where the auxiliary matrix $L_{m}$, defined by

$\mathrm{L}_{m}=\frac{\mathrm{j} k_{0}}{\pi} \triangle R \mathrm{H}^{T}(R) \mathrm{F}_{m}(R, R)$,

can be expressed as (compare with Eq. (122))

$\mathrm{L}_{m}=\mathrm{Q}_{m}^{21}(R, \Delta R)+\mathrm{Q}_{m}^{22}(R, \triangle R) \mathrm{K}_{m}$

In Step 4, the integral representation (123) in conjunction with Eqs. (130) and (133) implies that $\mathrm{T}_{m}(R-\Delta R)$ also satisfies Eq. (124).

In Step 5, we deduce from Eqs. (124) and (135) that $\mathrm{K}_{m}$ is given by Eq. (125) with $\mathrm{Q}_{m}^{22}(R, 2 \triangle R)$ and $\mathrm{Q}_{m}^{21}(R, 2 \triangle R)$ replaced by $\mathrm{Q}_{m}^{22}(R, \triangle R)$ and $\mathrm{Q}_{m}^{21}(R, \triangle R)$, respectively. Substituting this result in Eq. (132), we obtain the forward recurrence relation:

$$
\begin{aligned}
\mathrm{T}_{m}(R) & =\mathrm{Q}_{m}^{11}(R, \Delta R)+\left[\mathrm{I}+\mathrm{Q}_{m}^{12}(R, \Delta R)\right] \\
& \times\left[\mathrm{I}-\mathrm{T}_{m}(R-\triangle R) \mathrm{Q}_{m}^{22}(R, \Delta R)\right]^{-1} \\
& \times \mathrm{T}_{m}(R-\triangle R)\left[\mathrm{I}+\mathrm{Q}_{m}^{21}(R, \Delta R)\right] .
\end{aligned}
$$




\section{Appendix B}

The matrix equation (83) is of the form

$\left[\begin{array}{ll}A_{11} & A_{12} \\ A_{21} & A_{22}\end{array}\right]\left[\begin{array}{ll}B_{11} & B_{12} \\ B_{21} & B_{22}\end{array}\right]=\left[\begin{array}{cc}2 I-A_{11} & -A_{12} \\ -A_{21} & 2 I-A_{22}\end{array}\right]$,

where we have set

$\mathrm{A}=\left[\begin{array}{ll}\mathrm{A}_{11} & \mathrm{~A}_{12} \\ \mathrm{~A}_{21} & \mathrm{~A}_{22}\end{array}\right]=\mathrm{I}-\frac{\triangle R}{2} \mathrm{~A}_{0}(R)$.

An algorithm for solving Eq. (137) reads as

1. $X=A_{21} A_{11}^{-1}$,

2. $Y=X A_{12}-A_{22}$,

3. $B_{21}=-Y^{-1}\left(X A_{11}-2 X-A_{21}\right)$,

4. $B_{22}=-Y^{-1}\left(X A_{21}-A_{22}+2 I\right)$,

5. $B_{11}=-A_{11}^{-1}\left(A_{12} B_{21}+A_{11}-21\right)$,

6. $B_{12}=-A_{11}^{-1}\left(A_{12} B_{22}+A_{12}\right)$.

Thus, in the above algorithm, instead of computing the inverse of the $4 N_{\max } \times 4 N_{\max }$ matrix $\mathrm{B}$, we compute the inverses of the two $2 N_{\max } \times 2 N_{\max }$ matrices $\mathrm{A}_{11}$ and $\mathrm{Y}$.

\section{Appendix C}

In this appendix we prove the central recurrence relation (106) along with the representations (107) and (108). The proof of the forward recurrence relation is similar and is therefore omitted here.

Consider Eq. (105), that is,

$\mathrm{f}_{m}(R)=\mathrm{U}_{m}(R)\left[\mathrm{J}(r) \mathrm{a}_{m}+\mathrm{H}(R) \mathrm{s}_{m}^{1}\right]+2 \triangle R \mathrm{U}_{m}(R) \mathrm{G}(R, R) \mathrm{f}_{m}(R)$.

As in Appendix 1, we organize our derivation in several steps.

Step 1. Computing the integrals in Eqs. (101) and (103) with $R_{1}=R-\triangle R$ and $R_{2}=R+\Delta R$, by the midpoint quadrature formula, we obtain

$\mathrm{a}_{m}^{2}=2 \triangle R \frac{\mathrm{j} k_{0}}{\pi} \mathrm{H}^{T}(R) \mathrm{f}_{m}(R)$,

and

$\mathrm{s}_{m}=\mathrm{s}_{m}^{1}+2 \triangle R \frac{\mathrm{j} k_{0}}{\pi} \mathrm{J}^{T}(R) \mathrm{f}_{m}(R)$,

respectively. By substituting Eq. (140) in Eq. (102) with $\mathrm{T}_{m}^{1}=$ $\mathrm{T}_{m}(R-\triangle R)$, we get

$\mathrm{s}_{m}^{1}=\mathrm{T}_{m}(R-\triangle R) \mathrm{a}_{m}+2 \Delta R \frac{\mathrm{j} k_{0}}{\pi} \mathrm{T}_{m}(R-\Delta R) \mathrm{H}^{T}(R) \mathrm{f}_{m}(R)$,

while by substituting Eq. (142) in Eq. (141), we find

$\mathrm{s}_{m}=\mathrm{T}_{m}(R-\triangle R) \mathrm{a}_{m}$

$$
+2 \triangle R \frac{\mathrm{j} k_{0}}{\pi}\left[\mathrm{T}_{m}(R-\triangle R) \mathrm{H}^{T}(R)+\mathrm{J}^{T}(R)\right] \mathrm{f}_{m}(R) .
$$

Step 2. Inserting Eq. (142) in Eq. (139) yields the representation

$$
\begin{aligned}
\mathrm{f}_{m}(R) & =\left\{\mathrm{I}-2 \Delta R \mathrm{U}_{m}(R)\left[\mathrm{G}(R, R)+\frac{\mathrm{j} k_{0}}{\pi} \mathrm{H}(R) \mathrm{T}_{m}(R-\triangle R) \mathrm{H}^{T}(R)\right]\right\}^{-1} \\
& \times \mathrm{U}_{m}(R)\left[\mathrm{J}(r)+\mathrm{H}(R) \mathrm{T}_{m}(R-\triangle R)\right] \mathrm{a}_{m} .
\end{aligned}
$$

Step 3. From the equation $\mathrm{s}_{m}=\mathrm{T}_{m} \mathrm{a}_{m}$ with $\mathrm{T}_{m}=\mathrm{T}_{m}(R+\Delta R)$ on one hand, and Eq. (143) on the other hand, we obtain, in using Eq. (144),

$$
\begin{aligned}
& \mathrm{T}_{m}(R+\Delta R)=\mathrm{T}_{m}(R-\Delta R) \\
& \quad+2 \Delta R \frac{\mathrm{j} k_{0}}{\pi}\left[\mathrm{T}_{m}(R-\Delta R) \mathrm{H}^{T}(R)+\mathrm{J}^{T}(R)\right]
\end{aligned}
$$

$$
\begin{aligned}
& \times\left\{\mathrm{I}-2 \triangle R \mathrm{U}_{m}(R)\left[\mathrm{G}(R, R)+\frac{\mathrm{j} k_{0}}{\pi} \mathrm{H}(R) \mathrm{T}_{m}(R-\triangle R) \mathrm{H}^{T}(R)\right]\right\}^{-1} \\
& \times \mathrm{U}_{m}(R)\left[\mathrm{J}(r)+\mathrm{H}(R) \mathrm{T}_{m}(R-\triangle R)\right] .
\end{aligned}
$$

Step 4. In Eq. (145) we make the transformations (127), i.e., $\triangle R \rightarrow \frac{\triangle R}{2}$ and $R \rightarrow R-\frac{\triangle R}{2}$

and find the central recurrence relation:

$$
\begin{aligned}
\mathrm{T}_{m}(R) & =\mathrm{T}_{m}(R-\triangle R) \\
& +\Delta R \frac{\mathrm{j} k_{0}}{\pi}\left[\mathrm{T}_{m}(R-\Delta R) \mathrm{H}^{T}\left(R-\frac{\triangle R}{2}\right)+\mathrm{J}^{T}\left(R-\frac{\triangle R}{2}\right)\right] \\
& \times\left\{\mathrm{I}-\Delta R \mathrm{U}_{m}\left(R-\frac{\Delta R}{2}\right)\left[\mathrm{G}\left(R-\frac{\Delta R}{2}, R-\frac{\triangle R}{2}\right)\right.\right. \\
& \left.\left.+\frac{\mathrm{j} k_{0}}{\pi} \mathrm{H}\left(R-\frac{\triangle R}{2}\right) \mathrm{T}_{m}(R-\triangle R) \mathrm{H}^{T}\left(R-\frac{\Delta R}{2}\right)\right]\right\}^{-1} \\
& \times \mathrm{U}_{m}\left(R-\frac{\triangle R}{2}\right)\left[\mathrm{J}\left(R-\frac{\triangle R}{2}\right)+\mathrm{H}\left(R-\frac{\Delta R}{2}\right) \mathrm{T}_{m}(R-\Delta R)\right] .
\end{aligned}
$$

\section{Appendix D}

In this appendix we show that the recurrence relations (52) and (112) are identical. Parts of the proof are borrowed from Ref. [27].

1. Setting $\mathrm{T}_{m}=\mathrm{T}_{m}(R), \mathrm{T}_{m}^{1}=\mathrm{T}_{m}(R-\triangle R)$, and

$\left(\mathrm{I}-\mathrm{T}_{m}^{1} \mathrm{Q}_{m}^{22}\right)^{-1}=\mathrm{I}+\mathrm{A}$,

which yields

$\mathrm{A}=\mathrm{T}_{m}^{1} \mathrm{Q}_{m}^{22}\left(\mathrm{I}-\mathrm{T}_{m}^{1} \mathrm{Q}_{m}^{22}\right)^{-1}$,

and performing the matrix multiplications in Eq. (52), we obtain

$$
\begin{aligned}
T_{m} & =T_{m}^{1}+Q_{m}^{11}+T_{m}^{1} Q_{m}^{21}+Q_{m}^{12} T_{m}^{1}+Q_{m}^{12} T_{m}^{1} Q_{m}^{21} \\
& +A T_{m}^{1}+A T_{m}^{1} Q_{m}^{21}+Q_{m}^{12} A T_{m}^{1}+Q_{m}^{12} A T_{m}^{1} Q_{m}^{21} .
\end{aligned}
$$

2. Setting $\alpha=\mathrm{j} k_{0} / \pi$, and

$\left(\mathrm{I}-\alpha \mathrm{V}_{m} \mathrm{HT}_{m}^{1} \mathrm{H}^{T}\right)^{-1}=\mathrm{I}+\mathrm{B}$,

which gives

$\mathrm{B}=\alpha \mathrm{V}_{m} \mathrm{HT}_{m}^{1} \mathrm{H}^{T}\left(\mathrm{I}-\alpha \mathrm{V}_{m} \mathrm{HT}_{m}^{1} \mathrm{H}^{T}\right)^{-1}$,

we express the recurrence relation (112) as

$$
\begin{aligned}
\mathrm{T}_{m} & =\mathrm{T}_{m}^{1}+\mathrm{Q}_{m}^{11}+\mathrm{T}_{m}^{1} \mathrm{Q}_{m}^{21}+\mathrm{Q}_{m}^{12} \mathrm{~T}_{m}^{1}+\mathrm{T}_{m}^{1} \mathrm{Q}_{m}^{22} \mathrm{~T}_{m}^{1} \\
& +\alpha \mathrm{T}_{m}^{1} \mathrm{H}^{T} \mathrm{BV}_{m} \mathrm{~J}+\alpha \mathrm{T}_{m}^{1} \mathrm{H}^{T} \mathrm{BV}_{m} \mathrm{HT}_{m}^{1} \\
& +\alpha \mathrm{J}^{T} \mathrm{BV}_{m} \mathrm{~J}+\alpha \mathrm{J}^{T} \mathrm{BV}_{m} \mathrm{HT}_{m}^{1} .
\end{aligned}
$$

3. Using the matrix identity

$\mathrm{X}\left(\mathrm{I}_{m}-\mathrm{YX}\right)^{-1}=\left(\mathrm{I}_{n}-\mathrm{XY}\right)^{-1} \mathrm{X}$,

where in general, $X$ is an $n \times m$ matrix and $Y$ an $m \times n$ matrix, and taking into account the definition of the matrices $\mathrm{Q}_{m}^{i j}$ as given by Eqs. (54)-(57), we find the following identities:

$\alpha \mathrm{T}_{m}^{1} \mathrm{H}^{T} \mathrm{BV} \mathrm{J}_{m}=\mathrm{AT}_{m}^{1} \mathrm{Q}_{m}^{21}$,

$\alpha \mathrm{T}_{m}^{1} \mathrm{H}^{T} \mathrm{BV}_{m} \mathrm{HT}_{m}^{1}=\mathrm{AT}_{m}^{1}-\mathrm{T}_{m}^{1} \mathrm{Q}_{m}^{22} \mathrm{~T}_{m}^{1}$,

$\alpha \mathrm{J}^{T} \mathrm{BV}_{m} \mathrm{~J}=\mathrm{Q}_{m}^{12} \mathrm{~T}_{m}^{1} \mathrm{Q}_{m}^{21}+\mathrm{Q}_{m}^{12} \mathrm{AT}_{m}^{1} \mathrm{Q}_{m}^{21}$,

$\alpha \mathrm{J}^{T} \mathrm{BV}_{m} \mathrm{HT}_{m}^{1}=\mathrm{Q}_{m}^{12} \mathrm{AT}_{m}^{1}$

Substituting Eqs. (154)-(157) in Eq. (152) yields Eq. (149), and the proof is finished. 
It should be pointed out that a direct derivation of the matrix identities (154)-(157) can be obtained if we assume that the Neumann series for the inverse matrices in Eqs. (147) and (150) converge. Indeed, considering the Neumann series for the inverse $\left(\mathrm{I}-\mathrm{T}_{m}^{1} \mathrm{Q}_{m}^{22}\right)^{-1}$, i.e.,

$\left(\mathrm{I}-\mathrm{T}_{m}^{1} \mathrm{Q}_{m}^{22}\right)^{-1}=\mathrm{I}+\sum_{n=1}^{\infty}\left(\mathrm{T}_{m}^{1} \mathrm{Q}_{m}^{22}\right)^{n}$,

we deduce from Eq. (147) along with Eq. (57) that

$\mathrm{A}=\sum_{n=1}^{\infty}\left(\mathrm{T}_{m}^{1} \mathrm{Q}_{m}^{22}\right)^{n}=\sum_{n=1}^{\infty}\left(\alpha \mathrm{T}_{m}^{1} \mathrm{H}^{T} \mathrm{~V}_{m} \mathrm{H}\right)^{n}$.

Similarly, considering the Neumann series for $\left(\mathrm{I}-\alpha \mathrm{V}_{m} \mathrm{HT}_{m}^{1} \mathrm{H}^{T}\right)^{-1}$, i.e.,

$\left(\mathrm{I}-\alpha \mathrm{V}_{m} \mathrm{HT}_{m}^{1} \mathrm{H}^{T}\right)^{-1}=\mathrm{I}+\sum_{n=1}^{\infty}\left(\alpha \mathrm{V}_{m} \mathrm{HT}_{m}^{1} \mathrm{H}^{T}\right)^{n}$,

we find from Eq. (150) that

$\mathrm{B}=\sum_{n=1}^{\infty}\left(\alpha \mathrm{V}_{m} \mathrm{HT}_{m}^{1} \mathrm{H}^{T}\right)^{n}$.

Then, by making use of the identity

$\mathrm{T}_{m}^{1} \mathrm{H}^{T}\left(\alpha \mathrm{V}_{m} \mathrm{HT}_{m}^{1} \mathrm{H}^{T}\right)^{n}=\left(\alpha \mathrm{T}_{m}^{1} \mathrm{H}^{T} \mathrm{~V}_{m} \mathrm{H}\right)^{n} \mathrm{~T}_{m}^{1} \mathrm{H}^{T}$,

which can be proved by induction, we find for example that (cf. Eq. (154))

$$
\begin{aligned}
\alpha \mathrm{T}_{m}^{1} \mathrm{H}^{T} \mathrm{BV}_{m} \mathrm{~J} & \stackrel{(161)}{=} \sum_{n=1}^{\infty} \alpha \mathrm{T}_{m}^{1} \mathrm{H}^{T}\left(\alpha \mathrm{V}_{m} \mathrm{HT}_{m}^{1} \mathrm{H}^{T}\right)^{n} \mathrm{~V}_{m} \mathrm{~J} \\
\stackrel{(162)}{=} & \sum_{n=1}^{\infty}\left(\alpha \mathrm{T}_{m}^{1} \mathrm{H}^{T} \mathrm{~V}_{m} \mathrm{H}\right)^{n} \mathrm{~T}_{m}^{1}\left(\alpha \mathrm{H}^{T} \mathrm{~V}_{m} \mathrm{~J}\right) \\
& \stackrel{(56),(159)}{=} \mathrm{AT}_{m}^{1} \mathrm{Q}_{m}^{21} .
\end{aligned}
$$

\section{Appendix E}

The adaptive step-size procedure is standard in the numerical solution of differential equations and improves the numerical accuracy of the T matrix. At each step, the program receives the current value $R$, the computed value of $\mathrm{T}_{m}(R)$, and $\Delta R$, the last step size used. Using these data the program computes two approximations to $\mathrm{T}_{m}(R+\triangle R)$ : the first, $\mathrm{T}_{m}^{1}$, by application of the recurrence with step size $\Delta R$, and the second, $\mathrm{T}_{m}^{2}$, by application of the recurrence twice, with step size $\Delta R / 2$. A local error estimate is then obtained using the relative error norm $\delta=\left\|T_{m}^{1}-T_{m}^{2}\right\| / \Delta$, where $\Delta=\varepsilon\left\|\mathrm{T}_{m}^{2}\right\|$ is the absolute error norm, and $\varepsilon$ is a prescribed tolerance, e.g., $\varepsilon=10^{-4}$. If $\delta>2$, the step is rejected, and all calculations are repeated using a smaller step size $\Delta R_{\text {new }}$ obtained from

$\left(\frac{\triangle R_{\text {new }}}{\Delta R}\right)^{p+1}=\frac{1}{\delta}$

where $p$ is the order of the method. If $\delta<2$, the step is accepted, with the approximation $\mathrm{T}_{m}^{\mathrm{R}}$ to $\mathrm{T}_{m}(R+\Delta R)$ taken to be the Richardson extrapolated value

$\mathrm{T}_{m}^{\mathrm{R}}=\frac{2^{p}}{2^{p}-1} \mathrm{~T}_{m}^{2}-\frac{1}{2^{p}-1} \mathrm{~T}_{m}^{1}$.

The step size $\Delta R$ is passed on to the next step, unless $\delta<1 / 2$, in which case it is updated to $\Delta R_{\text {new }}$ via Eq. (163). The solution accuracy can be also improved by applying the recurrence twice with the constant steps $\Delta R$ and $\Delta R / 2$, and by employing the Richardson extrapolation rule on the iterated transition matrices $T_{m}^{1}$ and $T_{m}^{2}$.

\section{Appendix F}

In this appendix we describe the scaling procedure for the recurrence (52). If the expansion order $N$ is larger than a critical value $N_{c}$, and the size parameter is small, the Hankel functions explodes, and overflow errors in computing the matrix $Q_{m}^{22}$ occur. The value of $N_{\mathrm{c}}$ can be increased by using a normalized form representation of the Hankel functions, or more precisely, of the Neumann functions $y_{n}(k r)$. In view of the representations $j_{n}(k r)=$ $\bar{j}_{n}(k r) 10^{e_{\mathrm{J} n}}$ and $y_{n}(k r)=\bar{y}_{n}(k r) 10^{e_{\mathrm{Y} n}}$, where $e_{\mathrm{J} n}$ and $e_{\mathrm{Y} n}$ are the exponents of the Bessel and Neumann functions in decimal representation, respectively, we compute the fractional coefficients $\bar{j}_{n}(k r)$ and $\bar{y}_{n}(k r)$ by scaling appropriately the corresponding recurrence relations. Note that for large values of $n, e_{\mathrm{J} n}$ is a large negative integer, while $e_{\mathrm{Y} n}$ is a large positive integer; their sum $e_{\mathrm{J} n}+e_{\mathrm{Y} n}$ is however sufficiently small for the exponent field representation. The fractional coefficients are encapsulated in the matrices $\bar{J}_{n}(r)$ and $\overline{\mathrm{Y}}_{n}(r)$, and we write $J_{n}(r)=\bar{J}_{n}(r) \mathrm{E}_{\mathrm{J} n}$ and $\mathrm{Y}_{n}(r)=\overline{\mathrm{Y}}_{n}(r) \mathrm{E}_{\mathrm{Y} n}$, with $\mathrm{E}_{\mathrm{J} n}=10^{e_{\mathrm{J} n}} \mid$ and $\mathrm{E}_{\mathrm{Y} n}=10^{e_{\mathrm{Y}} \mid} \mid$. Extending $\bar{J}_{n}(r)$ and $\overline{\mathrm{Y}}_{n}(r)$ into the global diagonal matrices $\bar{J}(r)$ and $\bar{Y}(r)$, respectively, as well as, $E_{J n}$ and $E_{Y n}$ into the global matrices $E_{J}$ and $E_{Y}$, respectively, we compute the matrices

$\mathrm{Q}_{m}^{11}(R)=\mathrm{E}_{\mathrm{J}} \mathrm{Q}_{m}^{\mathrm{JJ}}(R) \mathrm{E}_{\mathrm{J}}$,

$\mathrm{Q}_{m}^{12}(R)=\mathrm{E}_{\mathrm{J}} \mathrm{Q}_{m}^{\mathrm{JJ}}(R) \mathrm{E}_{\mathrm{J}}+\mathrm{j}_{\mathrm{J}} \mathrm{Q}_{m}^{\mathrm{JY}}(R) \mathrm{E}_{\mathrm{Y}}$,

$\mathrm{Q}_{m}^{21}(R)=\mathrm{E}_{\mathrm{J}} \mathrm{Q}_{m}^{\mathrm{JJ}}(R) \mathrm{E}_{\mathrm{J}}+\mathrm{j}_{\mathrm{Y}} \mathrm{Q}_{m}^{\mathrm{YJ}}(R) \mathrm{E}_{\mathrm{J}}$,

$\widehat{\mathrm{Q}}_{m}^{22}(R)=\mathrm{E}_{\mathrm{J}} \mathrm{Q}_{m}^{\mathrm{JJ}}(R) \mathrm{E}_{\mathrm{J}} \mathrm{E}_{\mathrm{Y}}^{-1}-\mathrm{E}_{\mathrm{Y}} \mathrm{Q}_{m}^{\mathrm{YY}}(R)$

$$
+\mathrm{j}\left[\mathrm{E}_{\mathrm{J}} \mathrm{Q}_{m}^{\mathrm{JY}}(R)+\mathrm{E}_{\mathrm{Y}} \mathrm{Q}_{m}^{\mathrm{YJ}}(R) \mathrm{E}_{\mathrm{J}} \mathrm{E}_{\mathrm{Y}}^{-1}\right],
$$

where

$\mathrm{Q}_{m}^{\mathrm{J} J}(R)=\frac{\mathrm{j} k_{0}}{\pi} \overline{\mathrm{J}}^{T}(R) \mathrm{V}_{m}(R) \overline{\mathrm{J}}(R)$,

$\mathrm{Q}_{m}^{\mathrm{JY}}(R)=\frac{\mathrm{j} k_{0}}{\pi} \bar{J}^{T}(R) \mathrm{V}_{m}(R) \overline{\mathrm{Y}}(R)$,

$\mathrm{Q}_{m}^{\mathrm{YJ}}(R)=\frac{\mathrm{j} k_{0}}{\pi} \overline{\mathrm{Y}}^{T}(R) \mathrm{V}_{m}(R) \overline{\mathrm{J}}(R)$,

$\mathrm{Q}_{m}^{\mathrm{YY}}(R)=\frac{\mathrm{j} k_{0}}{\pi} \overline{\mathrm{Y}}^{T}(R) \mathrm{V}_{m}(R) \overline{\mathrm{Y}}(R)$.

Thus, instead of computing $Q_{m}^{22}$, which is susceptible to overflow errors (due to the presence of the term $E_{Y} Q_{m}^{Y Y} E_{Y}$ ), we compute $\widehat{Q}_{m}^{22}$ $\left(Q_{m}^{22}=\widehat{Q}_{m}^{22} E_{Y}\right)$ which is less susceptible to overflow errors (due to the presence of the terms $E_{\mathrm{Y}} Q_{m}^{\mathrm{YY}}$ and $\mathrm{E}_{\mathrm{Y}} Q_{m}^{\mathrm{YJ}} \mathrm{E}_{\mathrm{J}} E_{\mathrm{Y}}^{-1}$ ). The inverse matrix in Eq. (52) is then computed as

$\left[\mathrm{I}-\mathrm{T}_{m}(R-\triangle R) \mathrm{Q}_{m}^{22}\right]^{-1}=\mathrm{E}_{\mathrm{Y}}^{-1}\left[\mathrm{E}_{\mathrm{Y}}^{-1}-\mathrm{T}_{m}(R-\Delta R) \widehat{\mathrm{Q}}_{m}^{22}\right]^{-1}$.

The matrix $E_{Y}^{-1}$ in Eq. (167) may now lead to underflow, and so, to a singular matrix. However, the critical value at which underflow occurs is larger than before.

\section{References}

[1] Waterman PC. Matrix formulation of electromagnetic scattering. Proc IEEE 1965;53:805-12

[2] Waterman PC. Symmetry, unitarity and geometry in electromagnetic scattering. Phys Rev D 1971;3:825-39.

[3] Barber PW, Hill SC. Light scattering by particles: Computational methods. Singapore: World Scientific; 1990.

[4] Mishchenko MI, Travis LD, Lacis AA. Scattering, absorption and emission of light by small particles. Cambridge: Cambridge University Press; 2002.

[5] Lakhtakia A, Varadan VK, Varadan VV. Scattering by highly aspherical targets: EBCM coupled with reinforced orthogonalization. Appl Opt 1984;23:3502-9.

[6] Moroz A. Improvement of Mishchenko's T -matrix code for absorbing particles. Appl Opt 2005;44:3604-9.

[7] Petrov D, Shkuratov Y, Videen G. Optimized matrix inversion technique for the T-matrix method. Opt Lett 2007;32:1168-70.

[8] Kahnert M, Rother T. Modeling optical properties of particles with small-scale surface roughness: combination of group theory with a perturbation approach. Opt Express 2011;19:11138-51. 
[9] Somerville WRC, Auguié B, Le Ru EC. Severe loss of precision in calculations of T-matrix integrals. J Quant Spectrosc Radiat Transfer 2012;113:524-35.

[10] Somerville WRC, Auguié B, Le Ru EC. A new numerically stable implementation of the T-matrix method for electromagnetic scattering by spheroidal particles. J Quant Spectrosc Radiat Transfer 2013;123:153-68.

[11] Petrov D, Synelnyk E, Shkuratov Y, Videen G. The T-matrix technique for calculations of scattering properties of ensembles of randomly oriented particles with different size. J Quant Spectrosc Radiat Transfer 2006;102:85-110.

[12] Petrov D, Videen G, Shkuratov Y, Kaydash M. Analytic T-matrix solution of light scattering from capsule and bi-sphere particles: applications to spore detection. J Quant Spectrosc Radiat Transfer 2007;108:81-105.

[13] Petrov D, Shkuratov Y, Videen G. Sh-matrices method applied to light scattering by finite circular cylinders. J Quant Spectrosc Radiat Transfer 2008;109:1474-95.

[14] Petrov D, Shkuratov Y, Videen G. Application of Sh-matrix method to light scattering by prolate and oblate spheroids. J Opt 2010;12(9):095701.

[15] Petrov D, Shkuratov Y, Videen G. An analytical approach to electromagnetic wave scattering from particles of arbitrary shapes. J Quant Spectrosc Radiat Transfer 2011;112(11):1636-45.

[16] Mishchenko MI, Travis LD. T-matrix computations of light scattering by large spheroidal particles. Opt Commun 1994;109:16-21.

[17] Iskander MF, Lakhtakia A, Durney CH. A new procedure for improving the solution stability and extending the frequency range of the EBCM. IEEE Trans Antennas Propagat 1983;31:317-24.

[18] Lakhtakia A, Varadan VK, Varadan VV. Iterative extended boundary condition method for scattering by objects of high aspect ratios. J Opt Soc Am A 1984;76:906-12.

[19] Wall D. Methods of overcoming numerical instabilities associated with the Tmatrix method. In: Varadan VV, Varadan VK, editors. Acoustic, electromagnetic and elastic waves scattering - Focus on the T-matrix approach. New York: Pergamon Press; 1980. p. 269-86.
[20] Bates RHT, Wall DJN. Null field approach to scalar diffraction: I. general method; II. approximate methods; III. inverse methods. Phil Trans Roy Soc London 1977;287:45-117.

[21] Hackman RH. The transition matrix for acoustic and elastic wave scattering in prolate spheroidal coordinates. J Acoust Soc Am 1984;75:35-45.

[22] Doicu A, Wriedt T, Eremin Y. Light scattering by systems of particles. Berlin, Heidelberg, New York: Springer; 2006.

[23] Johnson BR. Invariant embedding T-matrix approach to electromagnetic scattering. Appl Opt 1988;27:4861-73.

[24] Bi L, Yang P, Kattawar GW, Mishchenko MI. Efficient implementation of the invariant embedding T-matrix method and the separation of variables method applied to large nonspherical inhomogenous particles. J Quant Spectrosc Radiat Transf 2013;116:169-83.

[25] Bi L, Yang P, Kattawar GW. Mishchenko MI. A numerical combination of extended boundary condition method and invariant embedding method applied to light scattering by large spheroids and cylinders. J Quant Spectrosc Radiat Transf 2013;123:17-22.

[26] Bi L, Yang P. Accurate simulation of the optical properties of atmospheric ice crystals with the invariant embedding T-matrix method. J Quant Spectrosc Radiat Transf 2014;138:17-35.

[27] Doicu A, Wriedt T. The invariant imbeeding T matrix approach. The Generalized Multipole Technique for light scattering. Wriedt T, Eremin Y, editors. Springer; 2018.

[28] Choi CH. A survey of numerical methods for solving matrix Riccati differential equations. Proc IEEE Conf Southeaston 1990;1:696-700.

[29] Davison EJ, Maki MC. The numerical solution of the matrix Riccati differential equation. IEEE Trans Automat Contr 1973;18:71-3.

[30] Kenney CS, Leipnik RB. Numerical integration of the differential matrix Riccati equation. IEEE Trans Automat Contr 1985;30:962-70.

[31] Leipnik RB. A canonical form and solution for the matrix Riccati differential equation. Bull Australian Math Soc 1986;26:355-61. 